FACTA UNIVERSITATIS

Series: Mechanical Engineering Vol. 15, N ${ }^{\mathrm{o}} 1,2017$, pp. 1 - 30

DOI: 10.22190/FUME170315001V

Original scientific paper

\title{
MULTILAYERED PLATE ELEMENTS WITH NODE-DEPENDENT KINEMATICS FOR THE ANALYSIS OF COMPOSITE AND SANDWICH STRUCTURES
}

\author{
UDC 539.3:624.01
}

\author{
Stefano Valvano, Erasmo Carrera \\ MUL2 group, Department of Mechanical and Aerospace Engineering, \\ Politecnico di Torino, Turin, Italy
}

\begin{abstract}
In this paper a new plate finite element (FE) for the analysis of composite and sandwich plates is proposed. By making use of the node-variable plate theory assumptions, the new finite element allows for a simultaneous analysis of different subregions of the problem domain with different kinematics and accuracy, in a global/local sense. In particular higher-order theories with an Equivalent-Single-Layer (ESL) approach are simultaneously used with advanced Layer-Wise (LW) models. As a consequence, the computational costs can be reduced drastically by assuming refined theories only in those zones/nodes of the structural domain where the resulting strain and stress states present a complex distribution. On the contrary, computationally cheaper, low-order kinematic assumptions can be used in the remaining parts of the plate where a localized detailed analysis is not necessary. The primary advantage of the present variable-kinematics element and related global/local approach is that no ad-hoc techniques and mathematical artifices are required to mix the fields coming from two different and kinematically incompatible adjacent elements, because the plate structural theory varies within the finite element itself. In other words, the structural theory of the plate element is a property of the FE node in this present approach, and the continuity between two adjacent elements is ensured by adopting the same kinematics at the interface nodes. According to the Unified Formulation by Carrera, the through-the-thickness unknowns are described by Taylor polynomial expansions with ESL approach and by Legendre polynomials with LW approach. Furthermore, the Mixed Interpolated Tensorial Components (MITC) method is employed to contrast the shear locking phenomenon. Several numerical investigations are carried out to validate and demonstrate the accuracy and efficiency of the present plate element, including comparison with various closed-form and FE solutions from the literature.
\end{abstract}

Key Words: Multilayered Plate Elements, Node-dependent Kinematics, Equivalent-Single-Layer, Global/Local Analysis, Layer-Wise

Received March 15, 2017 / Accepted April 02, 2017

Corresponding author: Stefano Valvano

Affiliation: Department of Mechanical and Aerospace Engineering, Politecnico di Torino, Corso Duca degli Abruzzi, 24, 10129 Torino, Italy

E-mail: stefano.valvano@polito.it 


\section{INTRODUCTION}

The development of new materials for advanced engineering applications leads to a complex analysis of layered structures in practice. This is mainly due to the complex anisotropy that characterizes this kind of structures and that leads to intricate mechanical phenomena. In some cases, structures may contain regions where three-dimensional (3D) stress fields occur. To accurately capture these localized 3D stress states, solid models or higher-order theories are necessary. The Finite Element Method (FEM) has a predominant role among the computational techniques implemented for the analysis of layered structures. The majority of FEM theories available in the literature are formulated by axiomatic-type theories. The conventional FEM plate model is the classical Kirchhoff-Love theory, and some examples are given in $[1,2]$, whose extension to laminates is known as the Classical Lamination Theory (CLT) [3]. Another classical plate element is based on the First-order Shear Deformation Theory (FSDT), which rely on the works by Reissner [4] and Mindlin [5]. To overcome the limitations of classical theories, a large variety of plate finite element implementations of higher-order theories (HOT) have been proposed in the last years. HOTbased $C^{0}$ finite elements $\left(C^{0}\right.$ means that the continuity is required only for the unknown variables and not for their derivatives) were discussed by Kant et al. [6] and Kant and Kommineni [7]. Many other papers are available in which HOTs have been implemented for plates, and more details can be found in the books by Reddy [8] and Palazotto and Dennis [9]. The HOT type theories presented are ESL models; the variables are independent of the number of layers. Differently the LW models permit to consider different sets of variables per each layer. Finite element implementations of Layer-Wise (LW) theories in the framework of axiomatic-type theories have been proposed by many authors, among which Noor and Burton [10], Reddy [11], Mawenya and Davies [12], Rammerstorfer et al. [13].

However, the high computational costs represent the drawback of refined plate theories or three-dimensional analyses. In recent years considerable improvements have been obtained towards the implementation of innovative solutions for improving the analysis efficiency for a global/local scenario. In this manner, the limited computational resources can be distributed in an optimal manner to study in detail only those parts of the structure that require an accurate analysis. In general, two main approaches are available to deal with a global/local analysis: refining the mesh or the FE shape functions in correspondence with the critical domain; formulating multi-model methods, in which different subregions of the structure are analyzed with different mathematical models. The coupling of a coarse mesh and a refined one can be addressed as single-theory or single-model methods, and many techniques are present in literature $[14,15,16]$. In general, multi-theory methods can be divided into sequential or multistep methods, and simultaneous methods. In a sequential multi-model, the global region is analyzed with an adequate model with a cheap computational cost to determine the displacement or force boundary conditions for a subsequent analysis at the local level. The local region can be modeled with a more refined theory, or it can be modeled with 3-D finite elements, see [17, 18, 19, 20]. The simultaneous multi-model methods are characterized by the analysis of the entire structural domain, where different subregions are modeled with different mathematical models and/or distinctly different levels of domain discretization, in a unique step. One of the simplest types of simultaneous multi-model methods for composite laminates analysis is the concept of selective ply grouping or sublaminates $[21,22,23]$. In the literature, the local region (i.e., the region 
where an accurate stress analysis is desired) is generally modeled by using 3-D finite elements in the domain of a selective ply grouping method. Recently, the authors have developed multi-model elements with variable through-the-thickness approximation by using 2-D finite elements for both local and global regions [24, 25, 26]. In this approach, the continuity of the primary variables between local and global regions was straightforwardly satisfied by employing Legendre polynomials. Another well-known method to couple incompatible kinematics in multi-model methods is the use of Lagrange multipliers, which serve as additional equations to enforce compatibility between adjacent subregions. In the three-field formulation by Brezzi and Marini [27], an additional grid at the interface is introduced. The unknowns are represented independently in each sub-domain and at the interface, where the matching is provided by suitable Lagrange multipliers. This method was recently adapted by Carrera et al. [28, 29, 30] to couple beam elements of different orders and, thus, to develop variable kinematic beam theories. Ben Dhia et al. [31, 32, 33, 34] proposed the Arlequin method to couple different numerical models by means of a minimization procedure. This method was adopted by $\mathrm{Hu}$ et al. [35, 36] for the linear and non-linear analysis of sandwich beams modeled via one-dimensional and two-dimensional finite elements, and by Biscani et al. [37] for the analysis of beams and by Biscani et al. [38] for the analysis of plates. Reddy and Robbins [39] and Reddy [40] presented a multiple-model method on the basis of a variable kinematic theory and on mesh superposition in the sense of Fish [41] and Fish and Markolefas [42]. Coupling was obtained by linking the FSDT variables, which are present in all the considered models, without using Lagrangian multipliers. The coupling of different kinematics model in the framework of composite beam structure, using the extended variational formulation (XVF), is presented in [43], sinus model and classical kinematics are coupled into non-overlapping domains. In the present work, a new simultaneous multiple-model method for $2 \mathrm{D}$ elements with node-dependent kinematics is developed. This node-variable capability enables one to vary the kinematic assumptions within the same finite plate element. The expansion order of the plate element is, in fact, a property of the FE node in the present approach. Therefore, between the finite elements, the continuity is ensured by adopting the same expansion order in the nodes at the element interface. This node-dependent finite element has been used by the authors in [44] using classical and HOT-type theories; Taylor polynomials were used with an ESL approach. The novelty of the present work lies in the combination of HOT-type and advanced LW theories in the same finite element. In this manner, global/local models can be formulated without using any mathematical artifice. As a consequence, computational costs can be reduced assuming refined models only in those zones with a quasi-three-dimensional stress field, whereas computationally cheap, low-order kinematic assumptions are used in the remaining parts of the plate structure. In this paper, the governing equations of the node-variable kinematic plate element for the linear static analysis of composite structures are derived from the Principle of Virtual Displacement (PVD). Subsequently, FEM is adopted and the Mixed Interpolation of Tensorial Components (MITC) method [45, 46, 47, 48] is used to contrast the shear locking. The developed methodology is, therefore, assessed and used for the analysis of multilayered cantilevered plates with concentrated loads, cross-ply plates with simply-supported edges and subjected to a localized pressure load, and asymmetric laminated sandwich plates simply-supported and subjected to a localized pressure load. The results are compared with various theories and, whenever possible, with exact solutions available from the literature. 


\section{REFINED AND HIERARCHICAL THEORIES FOR PLATES}

In this paper, different kinematic assumptions in different subregions of the problem domain are made by a new finite element which allows a simultaneous multi-model analysis, without ad-hoc techniques and mathematical artifices that are usually required to mix the fields coming from two different kinematic models. The present plate structural theory varies within the finite element itself.

To highlight the capabilities of the present formulation, a four-node plate element with node-dependent kinematics is shown in Fig. 1. The element proposed in this example makes use of a Layer-Wise theory of the first order at node 1. On the other hand, a second-order refined theory is employed at node 2 . At node 3 , a third order expansion is adopted. Finally, a Layer-Wise theory of the second order is assumed at node 4. As will be clear further in the paper, the choice of the nodal plate theory is arbitrary and node-variable kinematic plate elements will be used to implement multi-model methods for a global-local analysis. Before discussing the present formulation, a brief overview of the refined and advanced plate theories is given below or the sake of completeness. Plates are bi-dimensional structures in which one dimension (in general the thickness in the $z$ direction) is negligible with respect to the other two dimensions. The geometry and the reference system that are adopted throughout the present work are shown in Fig. 1.

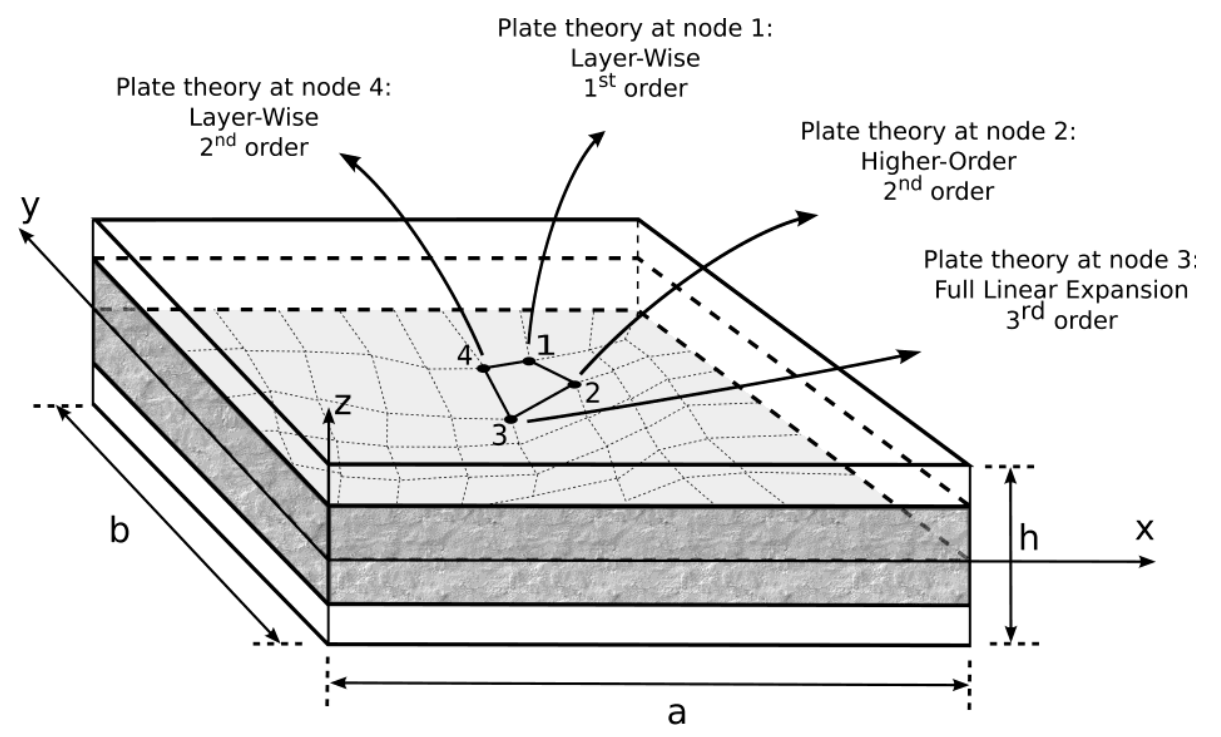

Fig. 1 Example of sandwich structure described by plate element with node-dependent kinematics

\subsection{Higher-Order Theories}

In order to overcome the limitations of classical theories, a large variety of plate higher-order theories (HOT) have been proposed in the past and recent literature. As a general guideline, it is clear that the richer the kinematics of the theory, the more accurate the 2D model becomes. HOT-type theories can be expressed by making use of Taylor-like 
expansions of the generalized unknowns along the thickness to formulate Equivalent-SingleLayer (ESL) models. In the case of generic expansions of $N$ terms, HOT displacement field can be expressed as in Eq. (1). For example, if a parabolic expansion order is taken into account, a graphical representation of a deflection can be depicted as in Fig. 2a. Moreover, Fig. 2b pictorially shows the capabilities of a generic HOT model, which can address complex kinematics in the thickness direction.

$$
\begin{aligned}
& u(x, y, z)=u_{0}(x, y)+z \quad u_{1}(x, y)+\ldots+z^{N} u_{N}(x, y) \\
& v(x, y, z)=v_{0}(x, y)+z v_{1}(x, y)+\ldots+z^{N} v_{N}(x, y) \\
& w(x, y, z)=w_{0}(x, y)+z w_{1}(x, y)+\ldots+z^{N} w_{N}(x, y)
\end{aligned}
$$

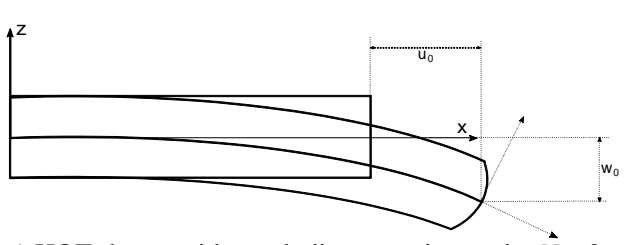

a) HOT-theory with parabolic expansion $\operatorname{order} \hat{N}=2$

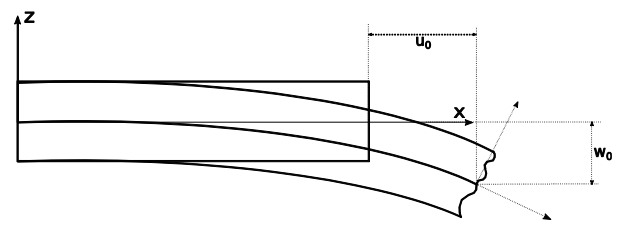

b) HOT-theory with generic expansion order

Fig. 2 Geometrical representation of the Higher-Order Theories

For the sake of completeness, the classical models, Classical Lamination Theory (CLT) $[1,2,3]$ and First-order Shear Deformation Theory (FSDT) [4, 5] kinematics, are particular cases of the full linear expansion, obtained from 1 imposing $N=1$. For more details see [49]. Therefore, it is well known in literature that linear models are affected by the problem of the Poisson Locking (PL) phenomenon. The remedy for the Poisson locking, apart from using higher-order theories, is to modify the Elastic Coefficients of the material. The PL phenomenon originates from constitutive laws which state the intrinsic coupling between inand out-of-plane strain components. Classical plate theories correct the locking phenomena by imposing that the out-of-plane normal stress is zero. This hypothesis yields reduced material stiff- ness coefficients which have to be accounted in the Hooke's law. Therefore, in literature, the correction of the material coefficients does not have a consistent theoretical proof. This means that the adoption of reduced material coefficients does not necessarily lead to the exact 3D solution, as shown in [50]. For the sake of clarity and simplicity of the present method explanation, the results presented in this work, with the full linear expansion kinematics, are not corrected for the PL phenomena.

\subsection{The Unified Formulation framework}

According to Unified Formulation by Carrera [49, 51, 52, 53], refined models can be formulated in a straightforward manner by assuming an expansion of each of the primary variables by arbitrary functions in the thickness direction. Thus, each variable can be treated independently of the others, according to the required accuracy. This procedure becomes extremely useful when multi-field problems are investigated such as thermoelastic and piezoelectric applications [54, 55, 56, 57]. In a displacement-based formulation, in fact, the three-dimensional displacement field is the combination of through-the-thickness functions weighted by the generalized unknown variables: 


$$
\begin{aligned}
& u(x, y, z)=F_{0}(z) u_{0}(x, y)+F_{1}(z) u_{1}(x, y)+\ldots+F_{N}(z) u_{N}(x, y) \\
& v(x, y, z)=F_{0}(z) v_{0}(x, y)+F_{1}(z) v_{1}(x, y)+\ldots+F_{N}(z) v_{N}(x, y) \\
& w(x, y, z)=F_{0}(z) w_{0}(x, y)+F_{1}(z) w_{1}(x, y)+\ldots+F_{N}(z) w_{N}(x, y)
\end{aligned}
$$

Similarly, in a compact form one has:

$$
\boldsymbol{u}(x, y, z)=F_{s}(z) \boldsymbol{u}_{s}(x, y) \quad s=0,1, \ldots, N
$$

where $\boldsymbol{u}(x, y, z)$ is the three-dimensional displacement vector, $\boldsymbol{u}(x, y, z)=[u, v, w] ; F_{S}$ are the thickness functions depending only on $z ; \boldsymbol{u}_{S}$ is the generalized displacement vector of the variables; $s$ is a sum index; and $N$ is the number of terms of the theory expansion. Depending on the choice of thickness functions $F_{S}$, and the number of terms in the plate kinematics $N$, various theories can be implemented.

\subsection{Advanced Theories}

The ESL models formulated with Taylor-like thickness functions, however, may not be sufficiently accurate to describe adequately the multilayered structures in which, due to their intrinsic anisotropy, the first derivative of the displacement variables in the $\mathrm{z}$ direction is discontinuous. Nevertheless, it is possible to reproduce the zig-zag effects in the ESL models by modifying opportunely $F_{S}$ functions, for example by adding the Murakami functions $[58,59]$. On the other hand, plate models with Layer-Wise (LW) capabilities can be implemented by describing the displacement components at the layer level, possibly by using a combination of Lagrange and Legendre-like polynomial as $F_{S}$ thickness functions $[60,61]$. In the case of Layer-Wise (LW) models, the displacement is defined at $k$-layer level:

$$
\begin{aligned}
& \boldsymbol{u}^{k}(x, y, z)=F_{t}(z) \boldsymbol{u}_{t}^{k}(x, y)+F_{b}(z) \boldsymbol{u}_{b}^{k}(x, y)+F_{r}(z) \boldsymbol{u}_{r}^{k}(x, y)=F_{s}(z) \boldsymbol{u}_{s}^{k}(x, y) \\
& s=t, b, r \quad r=2, \ldots, N \\
& F_{t}=\frac{P_{0}-P_{1}}{2} \quad F_{b}=\frac{P_{0}-P_{1}}{2} \quad F_{r}=P_{r}-P_{r-2}
\end{aligned}
$$

in which $P_{j}=P_{j}\left(\zeta_{k}\right)$ is the Legendre polynomial of $j$-order defined in the $\zeta_{k}$-domain: $-1 \leq \zeta_{k} \leq 1 ; P_{0}=1, P_{1}=\zeta_{k}, P_{2}=\left(3 \zeta_{k}^{2}-1\right) / 2, P_{3}=\left(5 \zeta_{k}^{3}-3 \zeta_{k}\right) / 2, P_{4}=\left(35 \zeta_{k}^{4}-30 \zeta_{k}^{2}+3\right) / 8$. The top $(t)$ and bottom $(b)$ values of the displacements are used as unknown variables and one can impose the following compatibility conditions:

$$
u_{t}^{k}=u_{b}^{k+1} \quad k=1, N_{l}-1
$$

For example, if a parabolic expansion order is taken into account, a graphical representation of a deflection can be depicted as in Fig. 3. 


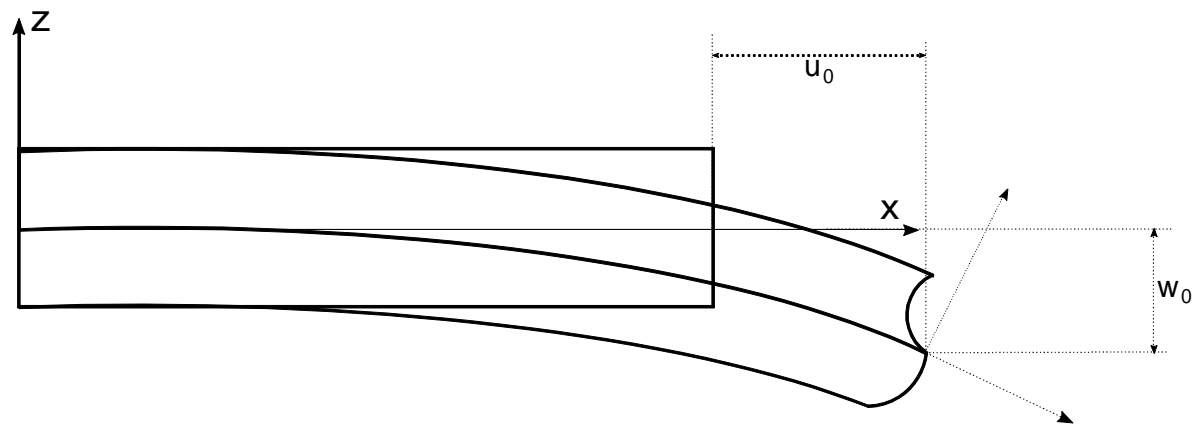

Fig. 3 Geometrical representation of a parabolic layer-wise model deflection on a two layered plate

\section{FINITE ELEMENT APPROXIMATION}

\subsection{Constitutive and geometrical relations for plates}

The definition of the 3D constitutive equations permits to express the stresses by means of the strains. The generalized Hooke's law is considered, by employing a linear constitutive model for infinitesimal deformations. In a composite material, these equations are obtained in material coordinates $(1,2,3)$ for each orthotropic layer $k$ and then rotated in the general reference system $(x, y, z)$. Therefore, the stress-strain relations after the rotation, calculated for each layer $k$, are:

$$
\boldsymbol{\sigma}^{k}=\boldsymbol{C}^{k} \boldsymbol{\varepsilon}^{k}
$$

where the stress and strain vectors have six components:

$$
\begin{aligned}
& \boldsymbol{\sigma}=\left[\sigma_{x x}, \sigma_{y y}, \sigma_{x y}, \sigma_{x z}, \sigma_{y z}, \sigma_{z z}\right] \\
& \boldsymbol{\varepsilon}=\left[\varepsilon_{x x}, \varepsilon_{y y}, \varepsilon_{x y}, \varepsilon_{x z}, \varepsilon_{y z}, \varepsilon_{z z}\right]
\end{aligned}
$$

and $\boldsymbol{C}$ is the material elastic coefficients matrix defined as follows:

$$
\boldsymbol{C}=\left[\begin{array}{cccccc}
C_{11} & C_{12} & C_{16} & 0 & 0 & C_{13} \\
C_{12} & C_{22} & C_{26} & 0 & 0 & C_{23} \\
C_{16} & C_{26} & C_{66} & 0 & 0 & C_{36} \\
0 & 0 & 0 & C_{55} & C_{45} & 0 \\
0 & 0 & 0 & C_{45} & C_{44} & 0 \\
C_{13} & C_{23} & C_{36} & 0 & 0 & C_{33}
\end{array}\right]
$$

For the sake of brevity, the expressions that relate material coefficients $C_{i j}$ to Young's moduli $E_{1}, E_{2}, E_{3}$, shear moduli $G_{12}, G_{13}, G_{23}$ and Poisson ratios $v_{12}, v_{13}, v_{23}, v_{21}, v_{31}, v_{32}$ are not given here. They can be found in many reference texts, such as [11].

The geometrical relations enable one to express strain vector $s$ in terms of displacement vector $\boldsymbol{u}$ for each layer $k$ : 


$$
\boldsymbol{\varepsilon}^{k}=\boldsymbol{D}_{g} \boldsymbol{u}^{k}
$$

where $\boldsymbol{D}_{g}$ is the geometrical vector containing the differential operators defined as follows:

$$
\boldsymbol{D}_{g}=\left[\begin{array}{cccccc}
\frac{\partial}{\partial x} & 0 & \frac{\partial}{\partial y} & \frac{\partial}{\partial z} & 0 & 0 \\
0 & \frac{\partial}{\partial y} & \frac{\partial}{\partial x} & 0 & \frac{\partial}{\partial z} & 0 \\
0 & 0 & 0 & \frac{\partial}{\partial x} & \frac{\partial}{\partial y} & \frac{\partial}{\partial z}
\end{array}\right]^{\mathrm{T}}
$$

\subsection{Node-dependant kinematics for plate finite elements}

By utilizing an FEM approximation, the generalized displacements of Eq. (3) can be expressed as a linear combination of the shape functions to have:

$$
\boldsymbol{u}_{\mathrm{s}}(x, y)=N_{j}(x, y) \boldsymbol{u}_{\mathrm{sj}} j=1, \ldots, \text { (nodes per element) }
$$

where $\boldsymbol{u}_{s j}$ is the vector of the generalized nodal unknowns, $N_{j}$ can be the usual Lagrange shape functions and $j$ denotes a summation on the element nodes. Since the principle of virtual displacements in used in this paper to obtain the elemental FE matrices, it is useful to introduce the finite element approximation of the virtual variation of generalized displacement vector $\delta \boldsymbol{u}_{\tau}$,

$$
\delta \boldsymbol{u}_{\tau}(x, y)=N_{i}(x, y) \delta \boldsymbol{u}_{\tau \mathrm{i}} \quad j=1, \ldots, \text { (nodes per element) }
$$

In Eq. (13), $\delta$ denotes the virtual variation, whereas indexes $\tau$ and $i$ are used instead of $s$ and $j$, respectively, for the sake of convenience.

In this work, and according to Eqs. (3), (12) and (13), thickness functions $F_{S}$ and $F_{\tau}$, which determine the plate theory order, are independent variables and may change for each node within the plate element. Namely, the three-dimensional displacement field and the related virtual variation can be expressed to address FE node-dependent plate kinematics as follows:

$$
\begin{aligned}
& \boldsymbol{u}(x, y, z)=F_{s}^{j}(z) N_{j}(x, y) \quad \boldsymbol{u}_{s j} \quad s=0,1, \ldots, N^{j} \quad j=1, \ldots, \text { (nodes per element) } \\
& \delta \boldsymbol{u}(x, y, z)=F_{\tau}^{j}(z) N_{j}(x, y) \delta \boldsymbol{u}_{s j} \quad \tau=0,1, \ldots, N^{j} i=1, \ldots, \text { (nodes per element) }
\end{aligned}
$$

where subscripts $\tau, s, i$, and $j$ denote summation. Superscripts $i$ and $j$ denote node dependency, such that for example $F_{\tau}^{i}$ is the thickness expanding function and $N^{i}$ is the number of expansion terms at node $i$, respectively. As example, the displacement field of the node-variable kinematic plate element as discussed in Fig. 1 is described in detail hereafter. The global displacement field of the element is approximated as follows:

- Node 1 Plate Theory = LW with $N^{1}=1$

- Node 2 Plate Theory $=$ HOT with $N^{2}=2$

- Node 3 Plate Theory $=$ HOT with $N^{3}=3$

- Node 4 Plate Theory $=$ LW with $N^{4}=2$

Eq. (4)

Eq. (1)

Eq. (1)

Eq. (4) 
According to Eq. (14), it is easy to verify that the displacements at a generic point belonging to the plate element can be expressed as given in Eq. (15). In this equation, only the displacement component along $x$-axis is given for simplicity reasons:

$$
\begin{aligned}
& u(x, y, z)=\left[\left(\frac{1+\zeta}{2}\right) u_{0_{1}}+\left(\frac{1-\zeta}{2}\right) u_{1_{1}}\right] N_{1}(x, y)+\left(u_{0_{2}}+z u_{1_{2}}+z^{2} u_{2_{2}}\right) N_{2}(x, y)+ \\
& +\left(u_{0_{3}}+z u_{1_{3}}+z^{2} u_{2_{3}}+z^{3} u_{3_{3}}\right) N_{3}(x, y)+\left[\left(\frac{1+\zeta}{2}\right) u_{0_{4}}+\left(\frac{1-\zeta}{2}\right) u_{1_{4}}+\left(\frac{3\left(\zeta^{2}-1\right)}{2}\right) u_{2_{4}}\right] N_{4}(x, y)
\end{aligned}
$$

It is intended that, due to node-variable expansion theory order, the assembling procedure of each finite element increases in complexity with respect to classical monotheory finite elements. In order to simplify the description of the assembling procedure, the governing equations are developed in form of fundamental nucleus, as described below.

\subsection{Governing equations and fundamental nucleus}

The governing equations for the static response analysis of the multi-layer plate structure can be obtained by using the principle of virtual displacements, which states:

$$
\int_{\Omega} \int_{A} \delta \varepsilon^{T} \boldsymbol{\sigma} d \Omega d z=\delta L_{e}
$$

where the term on the left-hand side represents the virtual variation of the strain energy; $\Omega$ and $A$ are the integration domains in the plane and the thickness direction, respectively; $\boldsymbol{\varepsilon}$ and $\sigma$ are the vector of the strain and stress components; and $\delta L_{e}$ is the virtual variation of the external loadings. By substituting the constitutive equations for composite elastic materials (Eq. (7)), the linear geometrical relations (Eq. 10) as well as Eq. (14) into Eq. (16), the linear algebraic system in the form of governing equations is obtained in the following matrix expression:

$$
\delta \boldsymbol{u}_{\tau_{i}}: \boldsymbol{K}^{\tau i j i} u_{s_{j}}=\boldsymbol{P}^{i i}
$$

where $\boldsymbol{K}^{\text {aij }}$ and $\boldsymbol{P}^{i i}$ are the element stiffness and load FE arrays written in the form of fundamental nuclei. The explicit expressions of the fundamental nuclei for node-dependent variable kinematic plate elements are given in [44]. It must be added that, in this study, an MITC technique is used to overcome the shear locking phenomenon, see [57]. The fundamental nucleus is the basic building block for the construction of the element stiffness matrix. In fact, given these nine components, element stiffness matrices of arbitrary plate models can be obtained in an automatic manner by expanding the fundamental nucleus versus indexes $\tau, s, i$, and $j$. In the present FE approach, the node-dependent variable kinematic model, it is clear that both rectangular and square arrays are handled and opportunely assembled for obtaining the final elemental matrices. In the development of ESL and LW theories, the fundamental nucleus of the stiffness matrix is evaluated at the layer level and then assembled as shown in Fig. 4. This figure, in particular, illustrates the expansion of the fundamental nucleus in the case of a 9-node Lagrange finite element with node-by-node variable kinematics, as in this paper. However, for more details about the explicit formulation of the Unified Formulation fundamental nuclei, interested readers are to refer to the recent book by Carrera et al. [49]. 


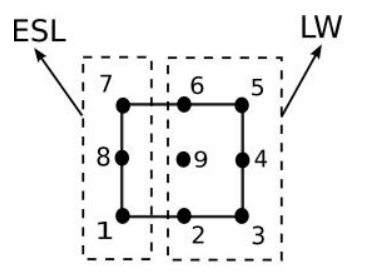

Assembling on the nodes $\mathrm{i}, \mathrm{j}$

j

i
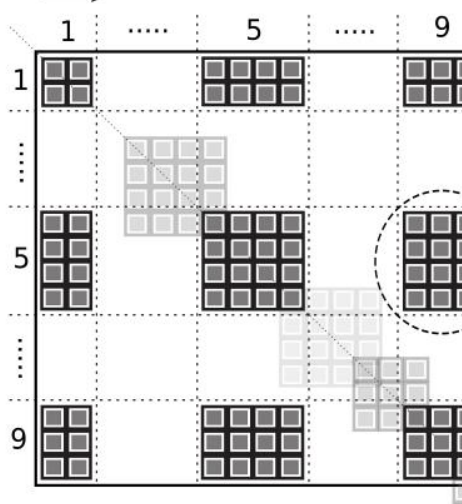
LW assembling other layers

$$
\begin{aligned}
& \mathbf{u}(x, y, z)=F_{S}^{j}(z) N_{j}(x, y) u_{\mathbf{s}} \quad \delta \mathbf{u}(x, y, z)=F_{\tau}^{i}(z) N_{j}(x, y) \delta u_{\boldsymbol{\tau}} \\
& S=0,1, \ldots, \mathcal{N}^{j} \quad \tau=0,1, \ldots, \mathcal{N}^{i} \quad i, j=1, \ldots, 9 \\
& \mathcal{N}^{\text {(node 1) }}=1 \cdots \cdots \mathcal{N}^{\text {(node 5) }}=3 \cdots \cdots \mathcal{N}^{\text {(node 9) }}=2
\end{aligned}
$$

Fig. 4 Assembling scheme of a 9-node finite element with node-dependent kinematics.

Highlights of the influence of the LW contribution of other layers in the FE stiffness

\section{NUMERICAL RESULTS}

In the numerical section some problems have been considered to assess the capabilities of the proposed node-variable kinematic plate elements and related global/local analysis. These cases of analysis comprise composite laminated and sandwich plate structures with different boundary conditions and loadings. Whenever possible, the proposed multi-theory models are compared to single-theory refined elements. The acronyms for the ESL models are indicated with the first letter $E$, the second letter indicates the polynomial kind, $T$ stands for Taylor polynomials and $L$ for Legendre polynomials. The LW models are indicated with the letters $L W$. The third letter indicates the number of the theory approximation order. If the analytical Navier solution type is employed, subscript $a$ is added. Moreover, analytical solution with higher-order single models, and multi-model theories present in literature are given for some cases. For the sake of clarity, present multi-model theories are opportunely described for each numerical case considered. 


\subsection{Eight-layer cantilever plate}

The first structure case taken into account is a simple example that easily permits to describe, through the results, the main capabilities of the present node-dependent plate element. A cantilever eight-layer plate is analyzed as shown in Fig. 5. The structure is loaded at the free end with a concentrated load equal to $P_{z}=-0.2 \mathrm{~N}$. The geometrical dimensions are: $a=90 \mathrm{~mm}, b=1 \mathrm{~mm}, h=10 \mathrm{~mm}$. The mechanical properties of the material labeled with the number 1 are: $E_{L}=30 \mathrm{GPa}, E_{T}=1 \mathrm{GPa}, G_{L T}=G_{T T}=0.5 \mathrm{GPa}, v_{L T}=v_{T T}=0.25$. On the other hand, the mechanical properties of the material labeled with the number 2 are: $E_{L}=$ $5 \mathrm{GPa}, E_{T}=1 \mathrm{GPa}, G_{L T}=G_{T T}=0.5 \mathrm{GPa}, v_{L T}=v_{T T}=0.25$. As it is clear from Fig. 5 , the material stacking sequence is $[1 / 2 / 1 / 2]_{s}$.

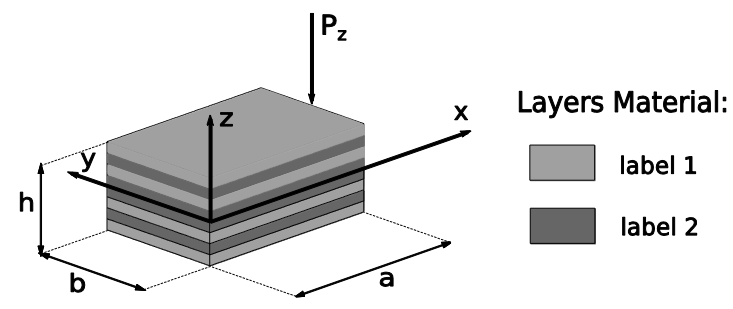

Fig. 5 Eight-layered plate with concentrated loading. Reference system and material lamination scheme

First, a convergence study on single-theory plate models was performed. For both LW4 and ET4 models, as shown in Table 1, a mesh grid of $12 \times 2$ elements is enough to ensure convergent results, for transverse mechanical displacement $w$, in-plane stress $\sigma_{x x}$ and transverse normal stress $\sigma_{x z}$. Various node-variable kinematic plate models have been used to perform the global/local analysis of the proposed plate structure, and they are depicted in Fig. 6. These models are compared in Table 2 with lower- to higher-order single-theory models as well as with various solutions from the literature, including an analytical solution based on the 2D elasticity as presented in Lekhnitskii [62].

It can be observed for transverse displacements $w$ that mono-theory LW models show a good accuracy solution independently of the polynomial order, differently for singlemodel ESL with Taylor polynomial yielding good results only with higher-order expansion ET3 and ET4. Moreover multi-theory ESL models Case A, Case B and Case C show an intermediate solution accuracy for all the three considered cases without relevant differences. For the multi-theory ESL-LW models Case D, Case E and Case F the solution is very accurate, due to the partial LW approximation, and exactly the same solution is obtained for the three considered cases.

Regarding in-plane stress $\sigma_{x x}$ the accuracy solution is not sensitive for all the considered single and multi model theories, except for the Case A configuration where the transition elements are acting at the evaluation position.

For transverse shear stress $\sigma_{x z}$ similar comments respect to the transverse displacement can be drawn. Single theory LW models show a good accuracy solution independently of the polynomial order, otherwise higher-order mono-model ESL theories with Taylor polynomial, ET3 and ET4, are required to obtain sufficient solution accuracy. Nevertheless, accurate solutions in localized regions/points can be obtained by using the multi-theory ESL model Case B, and with multi-theory ESL-LW models Case D and Case E. 

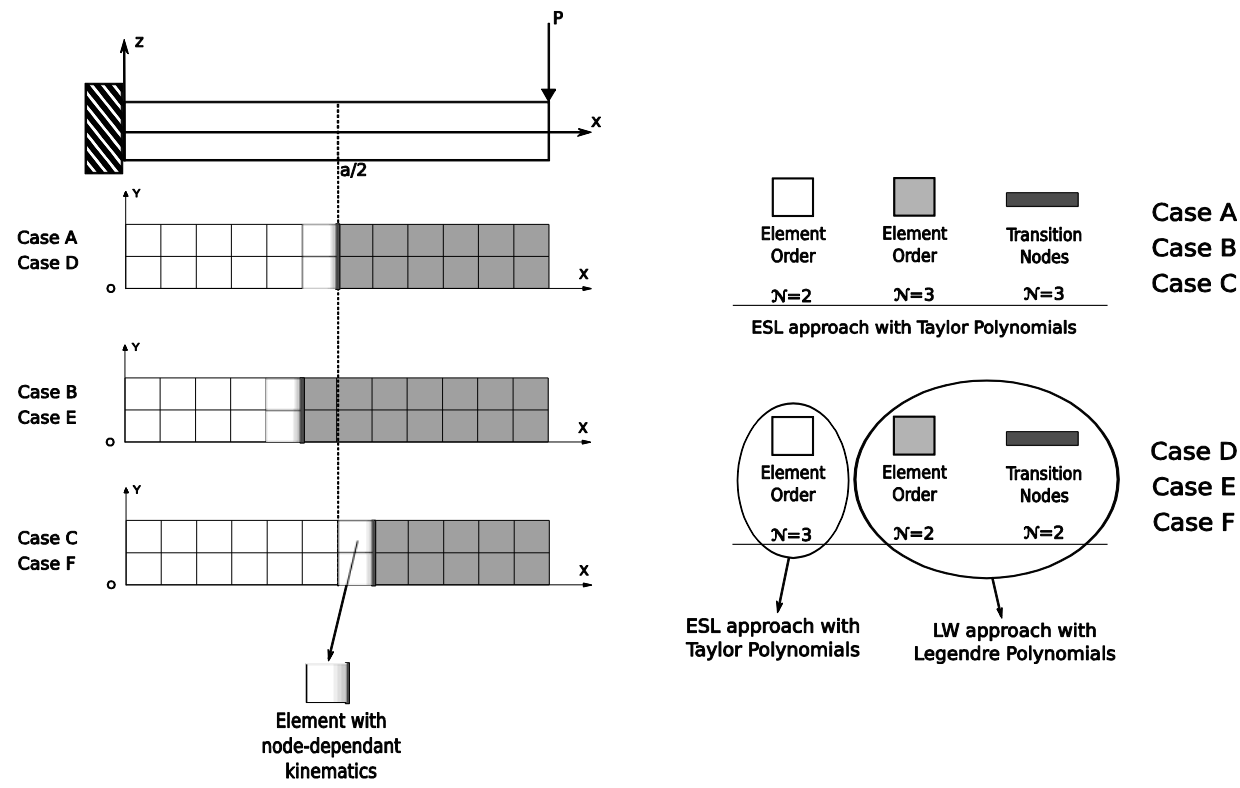

Fig. 6 Eight-layered plate. Mesh scheme of the adopted multi-theory models with node-dependent kinematics

Table 1 Convergence study of single-theory models of the eight-layer cantilever plate.

Transverse displacement $w=-10^{2} \times w(a, b / 2,0)$, in-plane principal stress $\sigma_{x x}=10^{3} \times \sigma_{x x}(a / 2, b / 2,+h / 2)$, transverse shear stress $\sigma_{x z}=-10^{2} \times \sigma_{x z}(a / 2, b / 2,0)$

\begin{tabular}{cccccccc}
\hline & Mesh & $2 \times 2$ & $4 \times 2$ & $6 \times 2$ & $8 \times 2$ & $10 \times 2$ & $12 \times 2$ \\
\hline \multirow{6}{*}{$L W 4$} & $w$ & 3.031 & 3.032 & 3.031 & 3.030 & 3.030 & 3.030 \\
& $\sigma_{x x}$ & 651 & 690 & 716 & 725 & 728 & 730 \\
& $\sigma_{x z}$ & 2.991 & 2.797 & 2.792 & 2.791 & 2.790 & 2.789 \\
$E T 4$ & $w$ & 3.029 & 3.029 & 3.029 & 3.028 & 3.028 & 3.028 \\
& $\sigma_{x x}$ & 684 & 723 & 729 & 730 & 731 & 731 \\
& $\sigma_{x z}$ & 3.054 & 2.829 & 2.820 & 2.821 & 2.822 & 2.822 \\
\hline
\end{tabular}

Some results in terms of transverse displacement $w$ and transverse shear stress $\sigma_{x z}$ along the thickness are represented in Figs. 7a and 7b, 8a and 8b, respectively. Some more comments can be made:

- As shown in Fig. 7a, the through-the-thickness distribution of transverse displacement $w$, evaluated at the free tip of the plate, is correctly predicted by a third-order ESL model ET3. The same accuracy cannot be reached by the proposed ESL models with node-variable kinematics. Differently, as depicted in Fig. 7b, both LW single theory and ESL-LW theory accuracy are not sensitive of the chosen model, except for the single linear model $L W 1$. 
Table 2 Eight-layer cantilever plate. Transverse displacement $\mathrm{w}=-10^{2} \times w(a, b / 2,0)$, in-plane normal stress $\sigma_{x x}=10^{3} \times \sigma_{x x}(a / 2, b / 2,+h / 2)$, transverse shear stress $\sigma_{x z}=-10^{2} \times \sigma_{x z}(a / 2, b / 2,0)$ by various single- and multi-theory models

\begin{tabular}{|c|c|c|c|c|}
\hline & $w$ & $\sigma_{x x}$ & $\sigma_{x z}$ & DOFs \\
\hline \multicolumn{5}{|c|}{ Reference solutions } \\
\hline Nguyen and Surana [63] & 3.031 & 720 & & \\
\hline Davalos et al. & 3.029 & 700 & & \\
\hline Xiaoshan [65] & 3.060 & 750 & & \\
\hline Vo and Thai [66] & 3.024 & & & \\
\hline Lekhnitskii [62] & & 730 & 2.789 & \\
\hline \multicolumn{5}{|c|}{ Present single- and multi-theory models } \\
\hline$L W 4$ & 3.030 & 730 & 2.789 & 12375 \\
\hline$L W 3$ & 3.030 & 731 & 2.788 & 9375 \\
\hline$L W 2$ & 3.030 & 731 & 2.795 & 6375 \\
\hline$L W 1$ & 3.022 & 731 & 2.775 & 3375 \\
\hline ET4 & 3.028 & 731 & 2.822 & 1875 \\
\hline ET3 & 3.027 & 731 & 2.822 & 1500 \\
\hline ET2 & 2.980 & 731 & 2.005 & 1125 \\
\hline$E T 1$ & 2.981 & 729 & 2.000 & 750 \\
\hline Case A & 3.004 & 808 & 2.375 & 1320 \\
\hline Case B & 3.010 & 737 & 2.781 & 1365 \\
\hline Case C & 3.002 & 731 & 2.030 & 1305 \\
\hline Case D & 3.028 & 732 & 2.799 & 4035 \\
\hline Case E & 3.028 & 729 & 2.799 & 4425 \\
\hline Case F & 3.028 & 731 & 2.818 & 3645 \\
\hline
\end{tabular}

- Figure 8a shows that transverse shear stress $\sigma_{x z}$, evaluated at the mid-span of the plate, is very sensitive to the position of the transition variable-kinematic elements. Case B model has the same accuracy as mono-model ET3 and ET4. On the contrary, the Case $C$ configuration has poor accuracy like mono-models ET1 and ET2. Finally, Case A model presents an intermediate compromise between the other two multitheory cases. All the ESL models are not able to reproduce the accurate behavior of the reference 2D elasticity solution Lekhnitskii, presented in [62]. On the contrary, as depicted in Fig. 8b, the LW single models are able to reach an accurate solution as the reference solution Lekhnitskii, except for the linear model $L W 1$. Multi-theory ESL-LW (ET3-LW2) models have a good approximation of the solution where the verification point is described by LW theories, Case $D$ and Case $E$ models, therefore Case $F$ show the same accuracy solution of model ET3.

By the evaluation of the various node-variable kinematic models, it is clear that an accurate representation of the stresses in localized zones is possible with DOFs reduction if an accurate distribution of the higher-order kinematic capabilities is performed in those localized zones. Differently, the displacements values are dependent on the global 
approximation over the whole structure. The DOFs reduction can be moderate or stronger, depending on the structure and the load case configuration.

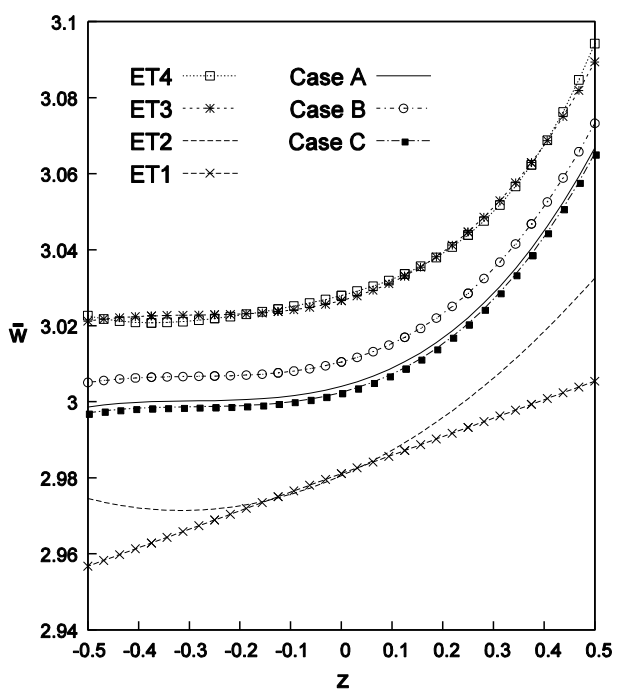

a) ESL single and multi model with Taylor Polynomials

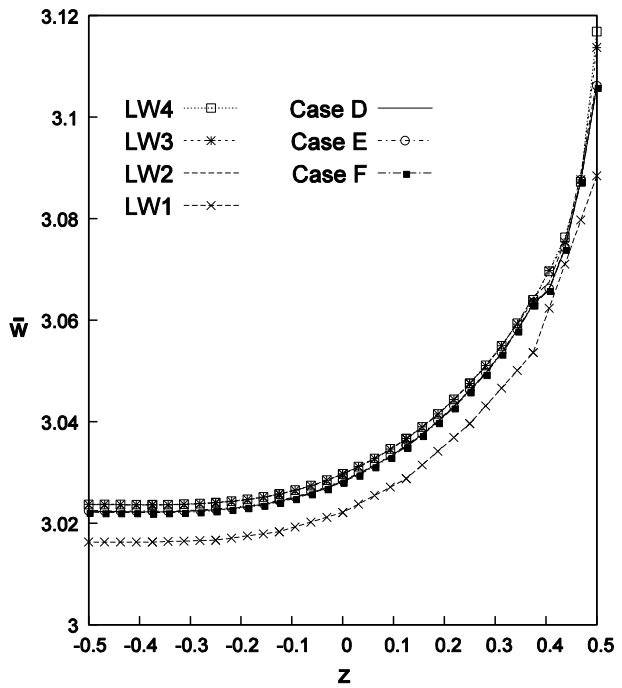

b) LW single model, and multi-theories with ESL model by Taylor Polynomials combined with LW model by Legendre Polynomials

Fig. 7 Eight-layer composite plate. Transverse displacement $w(x, y)=-10^{2} \times w(a, b / 2)$.

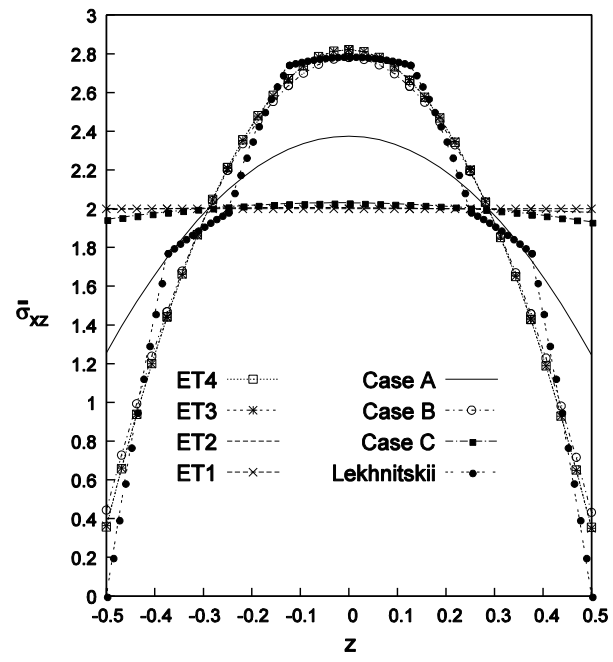

a) ESL single and multi model with Taylor Polynomials

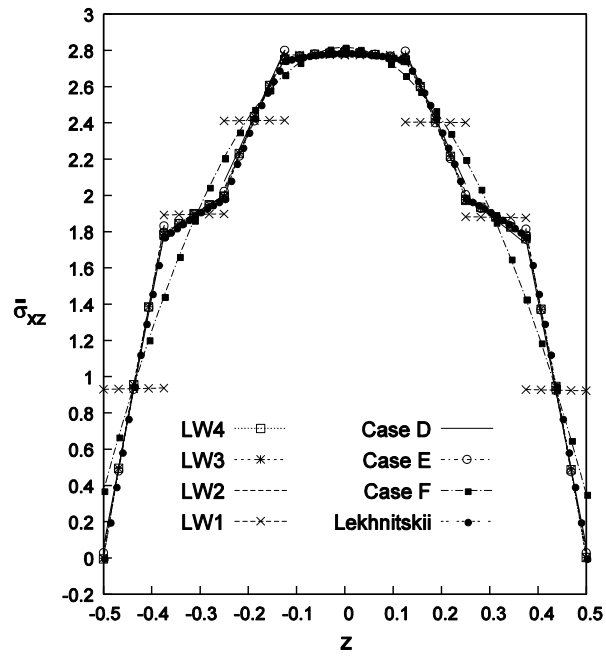

b) LW single model, and multi-theories with ESL model by Taylor Polynomials combined with LW model by Legendre Polynomials

Fig. 8 Eight-layer composite plate. Transverse displacement $\sigma_{x z}(x, y)=-10^{2} \times \sigma_{x z}(a / 2, b / 2)$ 


\subsection{Composite plates simply-supported}

A simply-supported composite plate is analyzed. The geometrical dimensions are: $a=b=$ $0.1 \mathrm{~m}$, the side-thickness ratio is $a / h=10$. A symmetric $\left[0^{\circ} / 90^{\circ} / 0^{\circ}\right]$ stacking sequences is considered. The material employed is orthotropic with the following properties: $E_{L}=132.5 \mathrm{GPa}$, $E_{T}=10.8 \mathrm{GPa}, G_{L T}=5.7 \mathrm{GPa}, G_{T T}=3.4 \mathrm{GPa}, v_{L T}=0.24, v_{T T}=0.49$. The plate is simplysupported and a localized uniform transverse pressure, $P_{Z}=-1 M P a$, is applied at top face on a square region of side length equal to $a / 5 \times b / 5$ and centered at the point $(a / 2, b / 2)$, see Fig. 9 . In order to take into account other solutions present in literature [38] a non-uniform mesh grid of $20 \times 20$ elements ensures the convergence of the solution, taken from [44], and it permits a fair comparison of the results. The non-uniform adopted mesh and the various node-variable kinematic models, with global/local capabilities used to perform the analysis of the proposed plate structure, are depicted in Fig. 9, where the mesh grid of a quarter of the plate is analyzed. Due to the symmetry of both the geometry and the load, a quarter of the plate is analyzed and the following symmetry and boundary conditions (simply-supported) are applied:

$$
\begin{aligned}
& \text { Boundary Symmetry } \\
& u_{s}(x, 0)=0 \quad w_{s}(x, 0)=0 \quad u_{s}(a / 2, y)=0 \\
& v_{s}(0, y)=0 \quad w_{s}(0, y)=0 \quad v_{s}(x, b / 2)=0
\end{aligned}
$$

The results are given in terms of transverse displacement $w$ and in-plane normal stresses $\sigma_{x x}, \sigma_{y y}$ evaluated at $(a / 2, b / 2,-h / 2)$, transverse shear stress $\sigma_{x z}$ evaluated at $(5 a / 12, b / 2,0)$, and transverse normal stress $\sigma_{z z}$ evaluated at $(a / 2, b / 2,+h / 2)$.

For the three-layered plate structure with $\left[0^{\circ} / 90^{\circ} / 0^{\circ}\right]$, mono-theory models are compared with those from the present global/local approach in Table 3. The table shows that mono-theory ESL models with lower expansion order, ET1 and ET2, are not able to describe appropriately transverse displacements $w$ and in-plane stresses $\sigma_{x x}$ and $\sigma_{y y}$; otherwise LW mono-models represent these variables with a good accuracy solution for every expansion order. To accurately describe shear transverse stresses $\sigma_{x z}$, ESL higherorder theories are required, or LW mono-models theories. Transverse normal stress $\sigma_{z z}$ needs higher-order theories to be well described; both linear ESL and LW single-models are not sufficient. Table 3 also shows solutions for variable kinematic multi-model theories; the cases taken into account are named from Case $A$ to $C a s e H$, and they are explained in Fig. 9. The cases named as Case A, Case B and Case E are equivalent to the models $(E T 1-E T 4)^{A},(E T 3-E T 4)^{B}$ and $(E T 1-L W 4)^{E}$ taken from [38] and in which, via the Arlequin method and 4-node Lagrangian plate elements, a fourth-order plate theory is used in correspondence of the loading; first- and third-order kinematics is used outside the loading zone, respectively. 


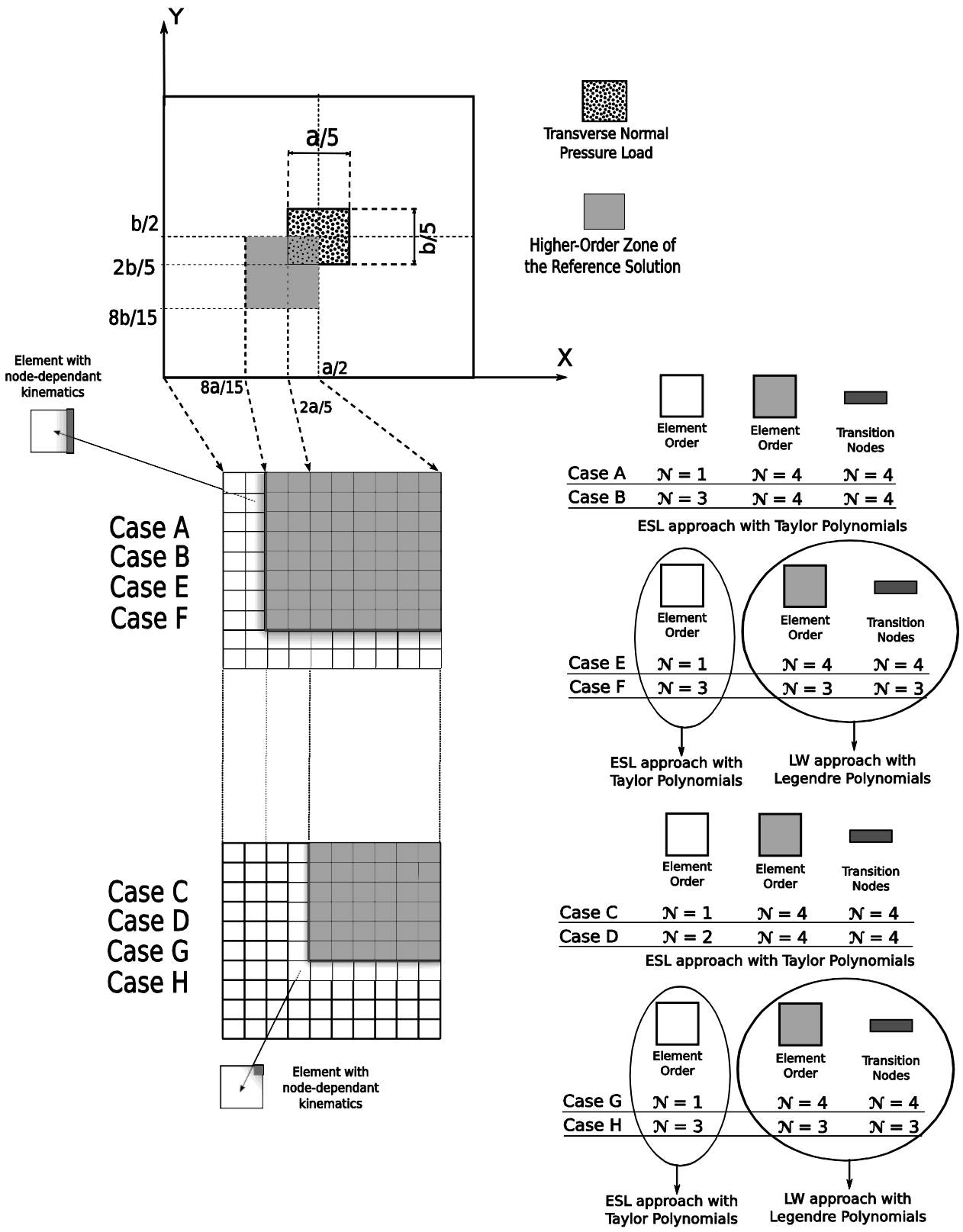

Fig. 9 Non-uniform adopted mesh on quarter of the plate, and graphical representation of the multi-theory models of the cross-ply plate structure 
Table 3 Composite plate with $\left[0^{\circ} / 90^{\circ} / 0^{\circ}\right]$ lamination. Transverse displacement $w=\left(-10^{5}\right) \times w(a / 2, b / 2,-h / 2)$, in-plane normal stresses $\sigma_{x x}=\sigma_{x x}(a / 2, b / 2,-h / 2)$ and $\sigma_{y y}=\sigma_{y y}(a / 2, b / 2,-h / 2)$, transverse shear stress $\sigma_{x z}=(-10) \times \sigma_{x z}(5 a / 12, b / 2,0)$, and transverse normal stress $\sigma_{z z}=-\sigma_{z z}(a / 2, b / 2,+h / 2)$ by various single- and multi-theory models

\begin{tabular}{|c|c|c|c|c|c|c|}
\hline & $w$ & $\sigma_{x x}$ & $\sigma_{v y}$ & $\sigma_{x z}$ & $\sigma_{z z}$ & DOFs \\
\hline \multicolumn{7}{|c|}{ Reference solutions [38] } \\
\hline $3 D$ & 1.674 & 11.94 & 2.019 & 6.524 & & \\
\hline$L W 4 a$ & 1.675 & 11.94 & 2.020 & 6.523 & & 39 \\
\hline$L W 4$ & 1.672 & 11.83 & 1.983 & 6.464 & & 9984 \\
\hline$E T 4_{a}$ & 1.660 & 11.95 & 2.005 & 5.865 & & 15 \\
\hline ET4 & 1.657 & 11.85 & 1.985 & 5.830 & & 3840 \\
\hline$(E T 1-E T 4)^{A}$ & 1.609 & 11.92 & 1.962 & 5.848 & & 2448 \\
\hline$(E T 3-E T 4)^{B}$ & 1.657 & 11.84 & 1.985 & 5.831 & & 3936 \\
\hline$(E T 1-L W 4)^{E}$ & 1.617 & 11.91 & 1.953 & 6.481 & & 3984 \\
\hline \multicolumn{7}{|c|}{ Present single- and multi-theory models } \\
\hline$L W 4$ & 1.6745 & 11.9547 & 2.0232 & 6.5557 & 1.0000 & 17199 \\
\hline$L W 3$ & 1.6745 & 11.9624 & 2.0302 & 6.5613 & 1.0108 & 13230 \\
\hline$L W 2$ & 1.6719 & 11.9141 & 2.0458 & 6.3903 & 1.0731 & 9261 \\
\hline$L W 1$ & 1.6369 & 11.3621 & 2.1465 & 6.5881 & 1.4679 & 5292 \\
\hline ET4 & 1.6596 & 11.9556 & 2.0078 & 5.8473 & 0.9905 & 6615 \\
\hline ET3 & 1.6590 & 11.9867 & 2.1164 & 6.0147 & 1.2443 & 5292 \\
\hline$E T 2$ & 1.5625 & 10.1942 & 1.7935 & 3.8521 & 1.0377 & 3969 \\
\hline ET1 & 1.4954 & 10.2867 & 2.1002 & 3.7554 & 1.8261 & 2646 \\
\hline Case A & 1.6040 & 12.0084 & 1.9821 & 5.8510 & 0.9910 & 5247 \\
\hline Case B & 1.6596 & 11.9556 & 2.0077 & 5.8473 & 0.9905 & 6159 \\
\hline Case C & 1.5257 & 11.7328 & 1.9453 & 4.9414 & 0.9938 & 4167 \\
\hline Case D & 1.5770 & 11.8056 & 1.9510 & 4.9970 & 0.9909 & 4983 \\
\hline Case E & 1.6103 & 12.0107 & 1.9923 & 6.5254 & 1.0000 & 12183 \\
\hline Case F & 1.6670 & 11.9699 & 2.0263 & 6.5524 & 1.0108 & 10494 \\
\hline Case $G$ & 1.5274 & 11.7105 & 1.9474 & 5.3212 & 1.0009 & 8223 \\
\hline Case H & 1.6613 & 11.9305 & 2.0198 & 6.3616 & 1.0118 & 8334 \\
\hline
\end{tabular}

Some results in terms of transverse displacement $w$, and transverse shear stress $\sigma_{x z}$ along the thickness are represented in Figs. 10a, 10b, 11a and 11b. The following remarks can be made:

- Transverse displacement $w$ behavior can change sensitively depending on the distribution of the kinematic enrichment within the structure plane. Figure 10a shows that Case B has the same accuracy as full higher-order ET 4 mono-model with a $8 \%$ DOFs reduction, and an accuracy close to multi-model Case $H$ with a $26 \%$ DOFs reduction. It is noticeable that the choice of the ESL or LW model for the loaded zone is not decisive for the correct description of the transverse mechanical displacement, as shown for Case $C$ and Case $G$. On the contrary a global more refined approximation get better accuracies, as in the case of the multi-models Case $A$ and Case E. 
- For the evaluation of transverse shear stress $\sigma_{x z}$, higher-order models are necessary in the regions close to the considered evaluation point. In Fig. 10b mono-model LW4 is used as a reference solution. It is evident that ESL single-models, for every expansion order, are not able to correctly describe the transverse shear stress. The
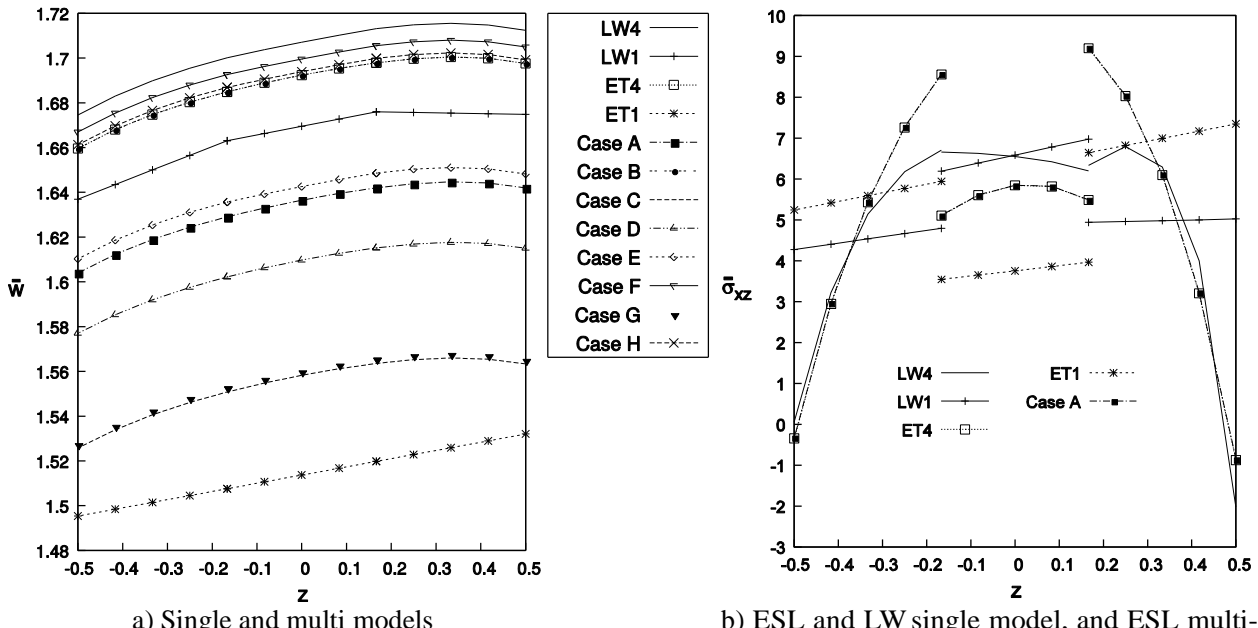

b) ESL and LW single model, and ESL multimodel with Taylor Polynomials

Fig. 10 Composite plate. Transverse displacement $w(x, y)=-10^{5} \times w(a / 2, b / 2)$, and transverse shear stress $\sigma_{x z}(x, y)=-10 \times \sigma_{x z}(5 a / 12, b / 2)$

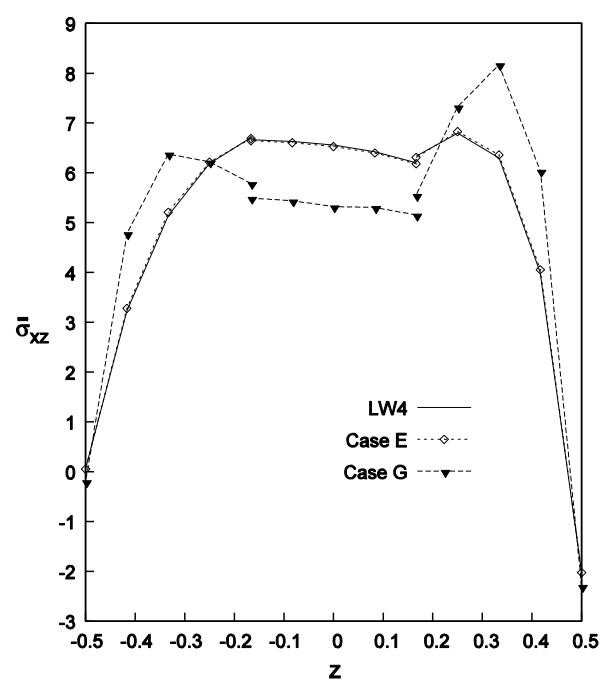

a)

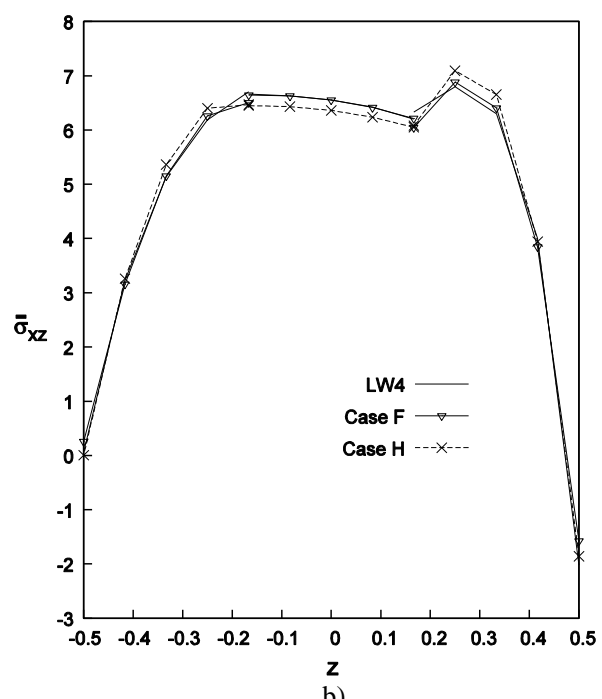

b)

Fig. 11 Composite plate. Transverse shear stress $\sigma_{x z}(x, y)=-10 \times \sigma_{x z}(5 a / 12, b / 2)$. Multi-theories with ESL model by Taylor Polynomials combined with LW model by Legendre Polynomials 
ESL multi-model Case A has the same poor accuracy of the theory ET4. Linear model $L W 1$ is clearly not sufficient to describe the transverse shear stress, differently from the single value reported in Table 3 taken in $z=0$. In Fig. 11a the multi-model Case E and Case $G$, where in the boundary regions a ET1 model is used and in the loaded zones a $L W 4$ model is adopted, the accuracy on the transverse shear stress is not completely guaranteed by the LW model. In particular for the Case $G$ the evaluation point is close to the transition element while this position is perturbation of the accuracy solution. On the contrary for the Case $E$ the evaluation point is not more close to the transition element and the solution accuracy is like the full LW model. Finally in Fig. $11 \mathrm{~b}$ the multi-model Case $F$ and Case $H$ are not suffering any perturbation problem, due to the third-order ESL model of the boundary regions.

Results in terms of in-plane stress $\sigma_{x x}$, transverse shear stress $\sigma_{x z}$ and transverse normal stress $\sigma_{z z}$ along the in-plane $x$-axis are represented in Figs. 12a, 12b and 13a, respectively.

For in-plane stress $\sigma_{x x}$, see Fig. 12a, the mono-models LW4 and ET4 show the same accuracy solution. Multi-models with ESL approach with Taylor polynomials, Case A and Case $C$, produce small oscillations in the transition zone. On the contrary, multi-theories with ESL model by Taylor Polynomials combined with LW model by Legendre Polynomials, Case $E$ and Case $G$, show big fluctuations in the transition elements. Moreover, it has to be noticed that if the refined polynomials are limited to the loading zone, Case $C$ and Case $G$, the solution accuracy in the loading zone is lower with respect to the reference $L W 4$ solution.

For transverse shear stress $\sigma_{x z}$, see Fig. 12b, the ET4 mono-model has an accuracy close to the mono-model LW4 in the loaded zone; differently the ET4 model reaches a maximum value of the shear stress for $9 \%$ lower than the reference $L W 4$ solution. For multi-model theories the same comments made for the in-plane stress can be applied for the behavior description of the transverse shear stress.

For transverse normal stress $\sigma_{z z}$, see Fig. 13a, mono-models LW4 and ET4 show the same accuracy solution. For multi-model theories the same comments made for the inplane stress can be applied for the behavior description of the transverse normal stress. It has to be noticed that the oscillations of the transition elements are smaller than those of the in-plane stress and the transverse shear stress.

Finally, a three-dimensional distribution of transverse shear stress $\sigma_{x z}$ is given on a quarter of the plate to underline the global/local capabilities of the present finite element on the whole domain of the analyzed plate structure. The reference single-model solution LW4 is depicted in Fig. 14a. For a fair comparison of the results, the extremes of the color bar values of the $L W 4$ model are used to limit the color bar of the other solutions. The single-model ET4 is not able to correctly describe the transverse shear stress behavior - it is clear from Fig. 14b that the interlaminar continuity of the transverse shear stress is not satisfied. In Fig. 15a the multi-model named Case E, (ET1-LW4) is represented. It is evident that the transverse shear stress is well represented in the $L W 4$ zone only. The multi-model Case H, (ET3-LW3) is represented in Fig. 15b, the small LW3 zone is able to correctly describe the transverse shear stress; on the contrary, the ET3 zone has a comparable behavior as the single-model ET4. 


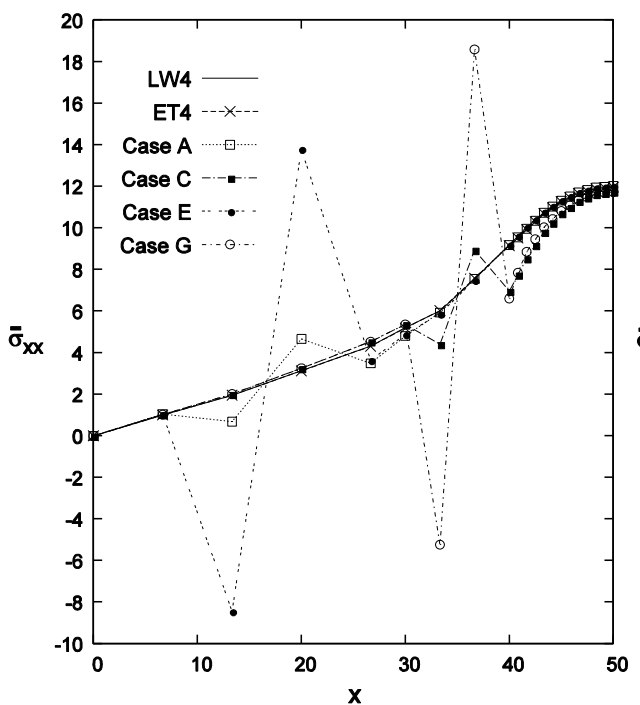

a) $\sigma_{x x}$

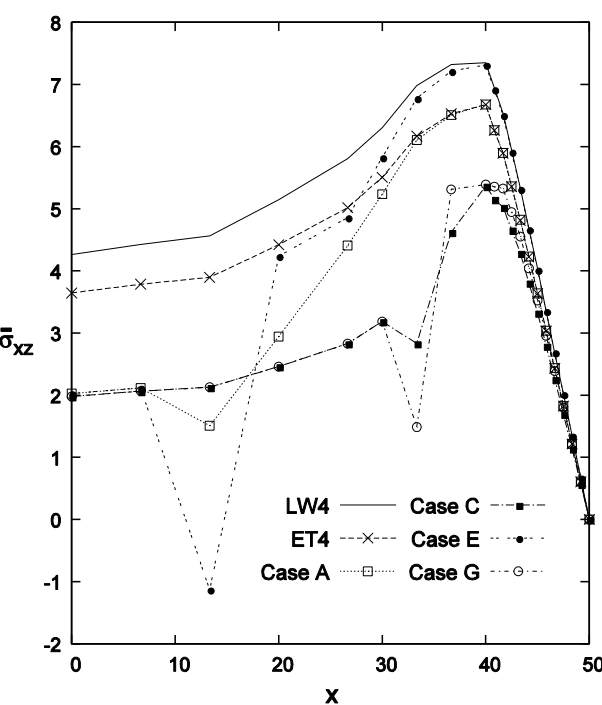

b) $\sigma_{x z}$

Fig. 12 Composite plate. In-plane stress $\sigma_{x x}(y, z)=\sigma_{x x}(b / 2,-h / 2)$, and transverse shear stress $\sigma_{x z}(y, z)=-10 \times \sigma_{x z}(b / 2,0)$ along the in-plane direction $x$, the $x$-axis is expressed in $[\mathrm{mm}]$. Single and Multi-theory models

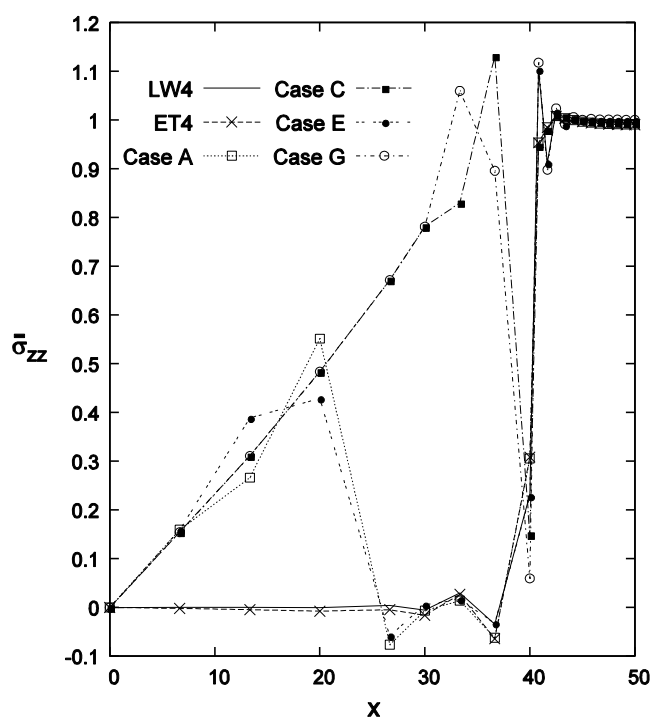

a) $\sigma_{z z}$

Fig. 13 Composite plate. Transverse normal stress $\sigma_{z z}(y, z)=-\sigma_{z z}(b / 2,+h / 2)$ along the inplane direction $x$, the $x$-axis is expressed in [mm]. Single and Multi-theory models 


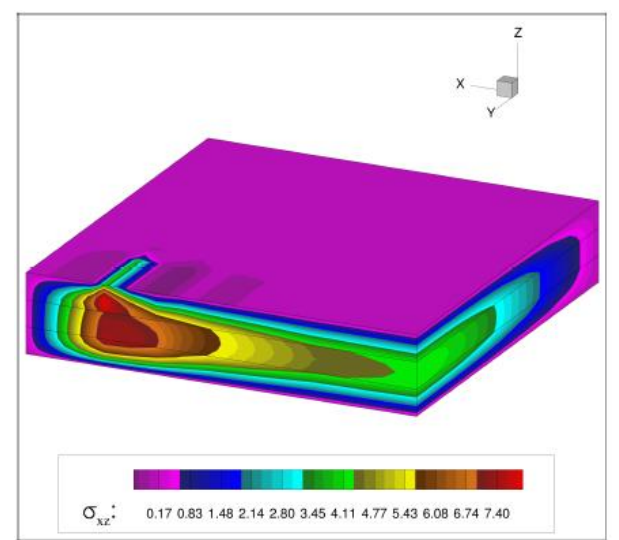

a) $L W 4$

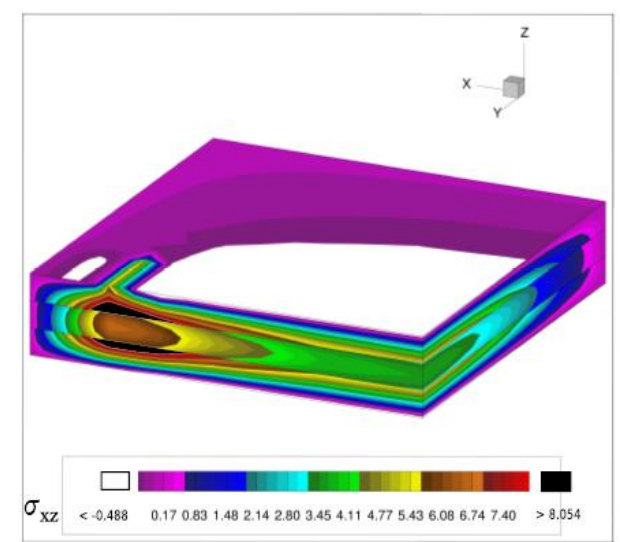

b) ET4

Fig. 14 Composite plate, three-dimensional view of a quarter of the plate. Transverse shear stress $\sigma_{x z}$ for single models

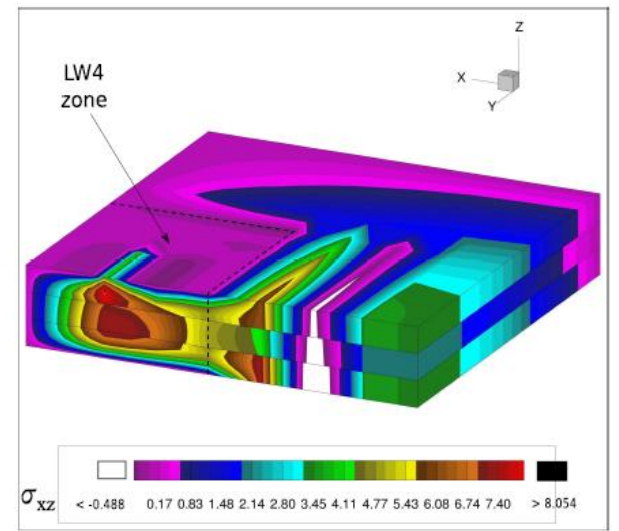

a) Case $\mathrm{E}$

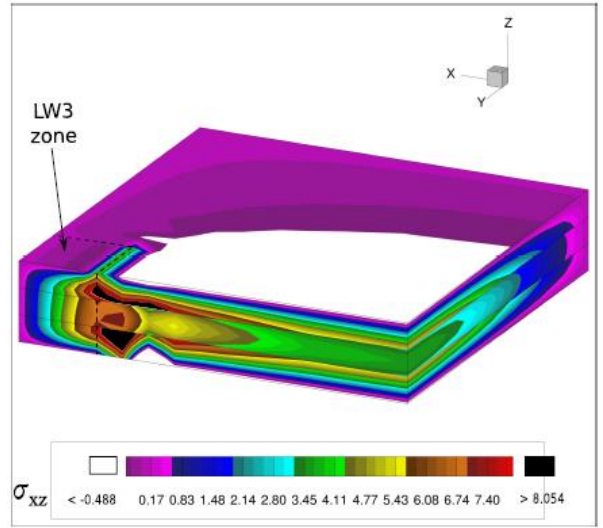

b) Case $\mathrm{H}$

Fig. 15 Composite plate, three-dimensional view of a quarter of the plate. Transverse shear stress $\sigma_{x z}$ for multi-models

\subsection{Sandwich rectangular plates simply-supported}

A simply-supported asymmetrically laminated rectangular sandwich plate is analyzed. The geometrical dimensions are: $a=100 \mathrm{~mm}, b=200 \mathrm{~mm}$, the total thickness is $h=12 \mathrm{~mm}$, the top skin thickness is $h_{\text {top }}=0.1 \mathrm{~mm}$, the bottom skin is thick $h_{\text {bottom }}=0.5 \mathrm{~mm}$, and the core thickness is $h_{\text {core }}=11.4 \mathrm{~mm}$. The two skins have the same material properties: $\quad E_{1}=70$ $G P a, E_{2}=71 G P a, E_{3}=69 G P a, G_{12}=G_{13}=G_{23}=26 G P a, v_{12}=v_{13}=v_{23}=0.3$, moreover, the metallic foam core has the following material properties: $E_{1}=E_{2}=3 \mathrm{MPa}, E_{3}=2.8$ $M P a, G_{12}=G_{13}=G_{23}=1 M P a, v_{12}=v_{13}=v_{23}=0.25$. The plate is simply-supported and localized uniform transverse pressure, $P_{Z}=-1 M P a$, is applied at top face on a square region of side length equal to $(a=5 \mathrm{~mm}) \times(b=20 \mathrm{~mm})$ and centered at the point $(a / 2, b / 2)$, see Fig. 16. 


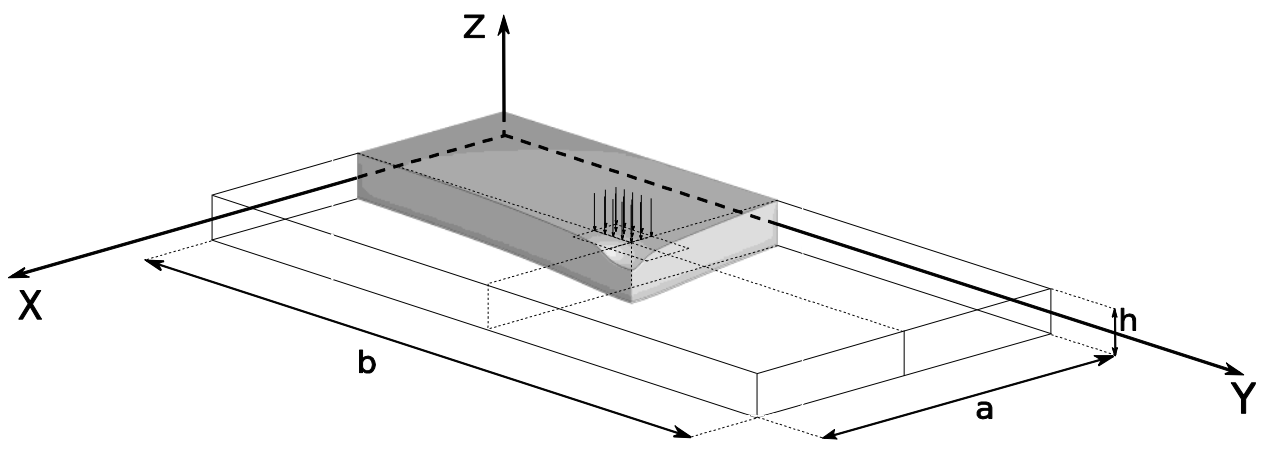

Fig. 16 Reference system of the sandwich plate. Three-dimensional deflection representation of a quarter of the plate

$$
\begin{array}{ccc}
\text { Boundary } & \text { Symmetry } \\
u_{s}(x, 0)=0 & w_{s}(x, 0)=0 & u_{s}(a / 2, y)=0 \\
v_{s}(0, y)=0 & w_{s}(0, y)=0 & v_{s}(x, b / 2)=0
\end{array}
$$

The present single- and multi-model solutions are compared with other solutions present in literature, three-dimensional analytical and three-dimensional FEM NASTRAN [67], ESL and LW analytical higher-order by the use of Fourier series expansions [68], ESL and LW FEM higher-order [69]. A non-uniform mesh grid of $38 \times 54$ elements ensures the convergence of the solution with a $L W 4$ single-model, see Fig. 17. For the sake of brevity the study of the convergence is here omitted. The adopted refined mesh is necessary to study the behavior of the mechanical variables along the whole plate domain, and not in one single point. The difficult task is to obtain a good behavior of the mechanical stresses, and in particular of transverse normal stress $\sigma_{z z}$ along the in-plane directions avoiding strange oscillations due to the changing of the element size.

For the asymmetrically laminated rectangular sandwich plate, mono-theory models are compared with those from the present global/local approach in Table 4. ESL models are not able to correctly describe all the variables; therefore, LW theories are necessary to match the reference analytical and 3D results. Table 4 also shows solutions for variable kinematic multi-model theories. As emerged in the previous numerical sections, the primary variables (displacements) depend on the global domain approximation, in particular transverse displacement $w$ is better described in the Case $B$ configuration with a DOFs reduction of $34 \%$ respect to the Case A multi-model. On the contrary, the postprocessed variables (stresses) are dependent on the local approximation. 

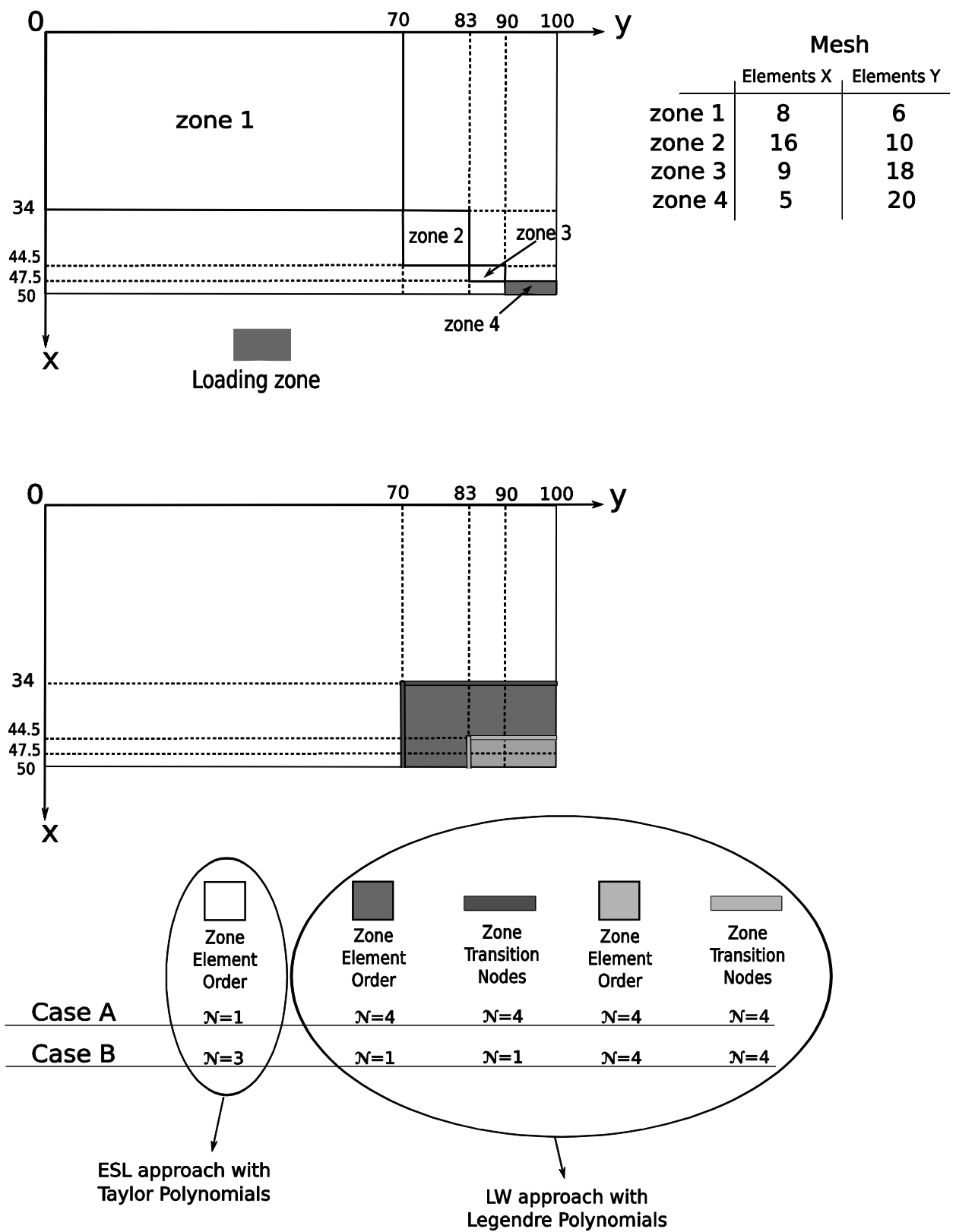

Fig. 17 Non-uniform adopted mesh and graphical representation of the multi-model cases, for a quarter of the sandwich plate 
Table 4 Asymmetrically laminated rectangular sandwich plate. Transverse displacement $w$, in-plane normal stresses $\sigma_{x x}$ and $\sigma_{y y}$, and transverse normal stress $\sigma_{z z}$ evaluated at $(a / 2, b / 2)$ by various single- and multi-theory models

\begin{tabular}{|c|c|c|c|c|c|c|}
\hline & $z$ & $w$ & $\sigma_{x x}$ & $\sigma_{y y}$ & $\sigma_{z z}$ & DOFs \\
\hline \multicolumn{7}{|c|}{ Top Skin } \\
\hline \multirow[t]{2}{*}{ 3D Analytical [67] } & Top & -3.78 & -624 & -241 & - & \\
\hline & Bottom & & 580 & 211 & - & \\
\hline \multirow[t]{2}{*}{ 3D NASTRAN [67] } & Top & -3.84 & -628 & -237 & - & \\
\hline & Bottom & & 582 & 102 & - & \\
\hline \multirow[t]{2}{*}{ LWM2 Analytical [68] } & Top & -3.8243 & -619.49 & - & - & \\
\hline & Bottom & & 577.36 & - & - & \\
\hline \multirow[t]{3}{*}{ LWM2 FEM [69] } & Top & -3.7628 & -595.56 & -223.93 & - & \\
\hline & Bottom & & 556.00 & 196.37 & - & \\
\hline & \multicolumn{6}{|c|}{ Top Skin } \\
\hline \multirow[t]{2}{*}{$L W 4$} & Top & -3.7774 & -622.48 & -233.39 & -0.9649 & 327327 \\
\hline & Bottom & & 578.60 & 203.25 & -0.8738 & \\
\hline \multirow[t]{2}{*}{$L W 3$} & Top & -3.7723 & -618.14 & -232.33 & -1.0143 & 251790 \\
\hline & Bottom & & 574.87 & 202.36 & -0.8270 & \\
\hline \multirow[t]{2}{*}{$L W 2$} & Top & -3.7552 & -601.46 & -228.13 & -0.9813 & 176253 \\
\hline & Bottom & & 559.72 & 198.73 & -0.8710 & \\
\hline \multirow[t]{2}{*}{$L W 1$} & Top & -3.3896 & -562.86 & -286.15 & -242.69 & 100716 \\
\hline & Bottom & & 530.98 & 262.78 & 240.82 & \\
\hline \multirow[t]{2}{*}{ ET4 } & Top & -2.5498 & -248.99 & -38.930 & 256.87 & 125895 \\
\hline & Bottom & & 184.89 & -1.7709 & -275.80 & \\
\hline \multirow[t]{2}{*}{ ET3 } & Top & -0.5995 & -121.19 & -56.428 & -21.706 & 100716 \\
\hline & Bottom & & 59.439 & 8.9946 & -19.349 & \\
\hline \multirow[t]{2}{*}{$E T 2$} & Top & -0.0238 & -29.573 & -28.178 & -30.655 & 75537 \\
\hline & Bottom & & -27.989 & -27.470 & -29.934 & \\
\hline \multirow[t]{2}{*}{$E T 1$} & Top & -0.0191 & -29.740 & -25.448 & -25.404 & 50358 \\
\hline & Bottom & & -29.444 & -25.211 & -25.248 & \\
\hline \multirow[t]{2}{*}{ Case A } & Top & -2.1386 & -622.21 & -220.95 & -0.9649 & 245619 \\
\hline & Bottom & & 567.44 & 198.35 & -0.8738 & \\
\hline \multirow[t]{2}{*}{ Case B } & Top & -2.4177 & -609.14 & -217.40 & -0.9654 & 161007 \\
\hline & Bottom & & 563.79 & 196.16 & -0.8663 & \\
\hline
\end{tabular}

Some results in terms of transverse displacement $w$, and transverse normal stress, $\sigma_{z z}$, along the thickness of the sandwich plate are represented in Figs. 18a, and 18b. The transverse displacement $w$ behavior can change sensitively depending on the distribution of the kinematic enrichment within the structure plane. Fig. 18a shows that ESL monomodels can vary sensitively their accuracy depending on the expansion order; differently the LW mono-models have almost the same accuracy independently of the adopted expansion. Moreover, for the multi-models, it is noticeable that the choice of the LW higher-order models for the loaded zone is not decisive for the correct description of the transverse mechanical displacement, as shown for Case A and Case B.

On the other hand, for transverse normal stress $\sigma_{z z}$, see Fig. 18b, LW higher-order models are able to correctly predict a good behavior along the plate thickness. Multi- 
models theories Case A and Case B show the same accuracy of the reference solution LW4 in the considered evaluation point.

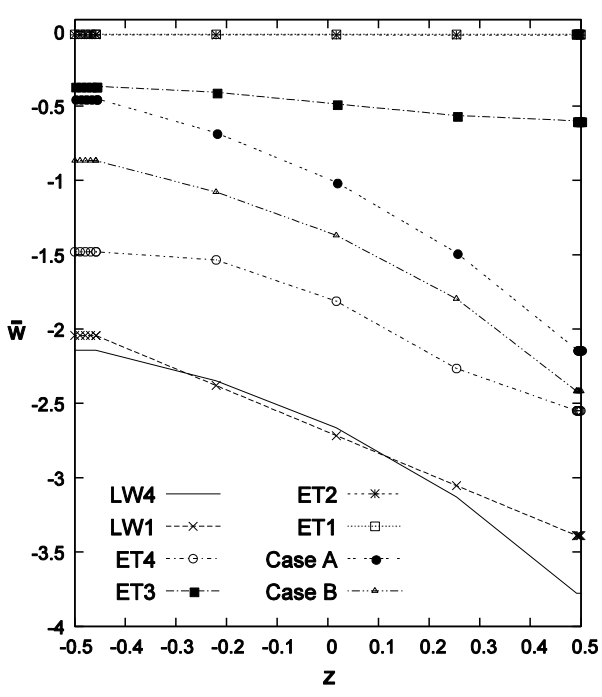

a) $w$

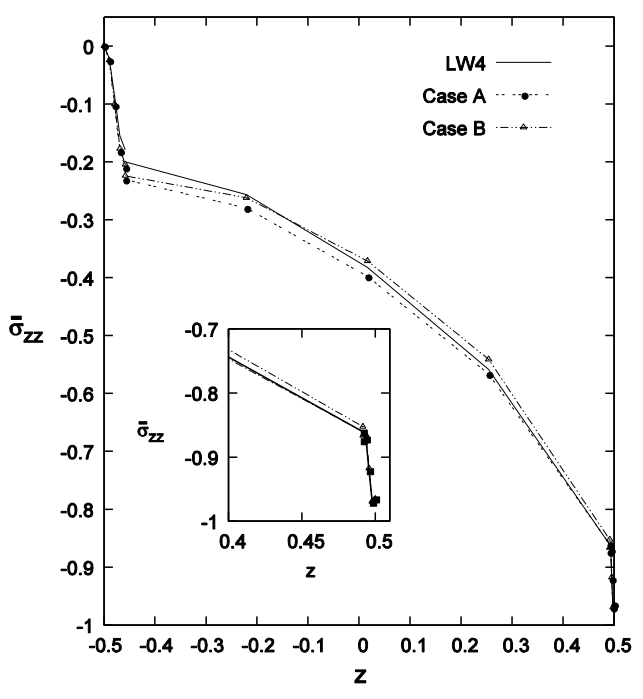

b) $\sigma_{z z}$

Fig. 18 Unsymmetrically laminated rectangular sandwich plate. Transverse displacement $w(x, y)$, and transverse normal stress $\sigma_{z z}(x, y)$ evaluated at $(a / 2, b / 2)$ by various single- and multi-theory models

Results in terms of the three-dimensional representation of in-plane stress $\sigma_{x x}$ and its behavior along the in-plane $x$ axis are represented in Figs. 19a and 19b, respectively. In Fig. 19a it is noticeable that the maximum values of the in-plane stress are located in the loading zone and its surroundings. Furthermore, the behavior of in-plane stress $\sigma_{x x}$ along the in-plane $x$ axis and evaluated at $(y, z)=(b / 2,+h / 2)$ is depicted in Fig. 19b. Monomodels LW4 and ET4 and multi-models Case $A$ and Case $B$ show almost the same accuracy solution. Multi-models Case $A$ and Case B, produce small oscillations in the transition zone. It is noticeable that the oscillations are small. This is due to a finer mesh with respect to the case of the previous numerical section.

Finally, a three-dimensional distribution on a quarter of the sandwich plate of transverse normal stress $\sigma_{z z}$ is given to underline the global/local capabilities of the present finite element on the whole domain of the analyzed sandwich plate structure. Referential singlemodel solution LW4 is depicted in Fig. 20a. For a fair comparison of the results, the extremities of the color bar values of the $L W 4$ model are used to limit the color bar of the other solutions. The single-model ET4 is not able to correctly describe the transverse shear stress behavior, as shown in Fig. 20b. 


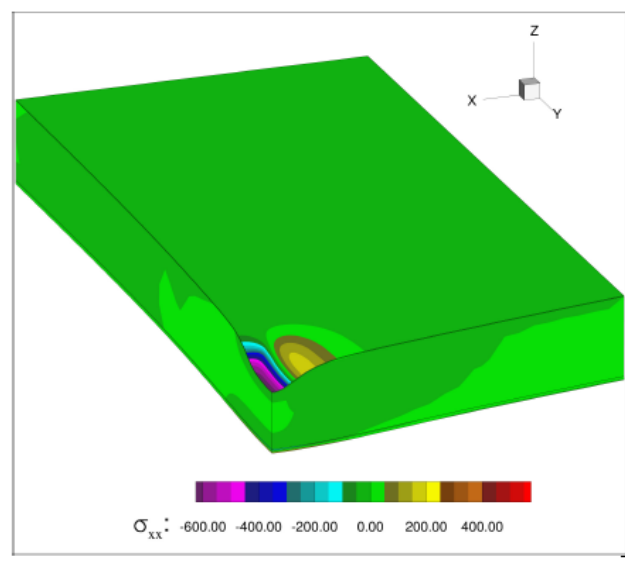

a) $L W 4$

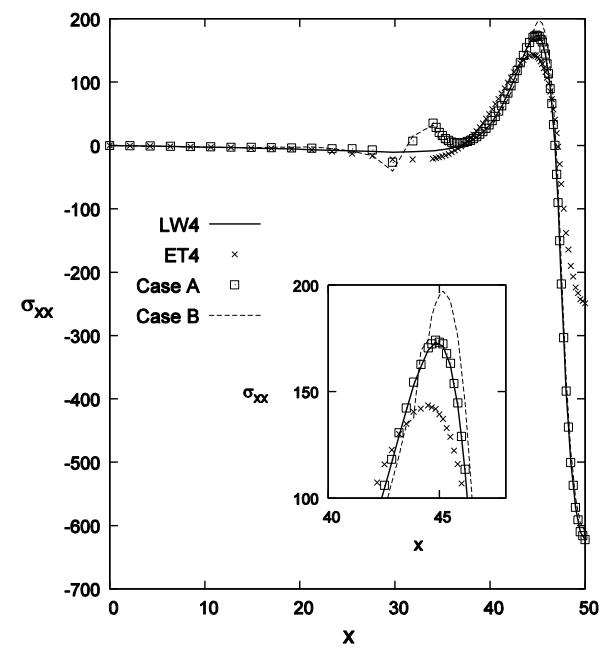

b) Single and multi-models

Fig. 19 Asymmetrically laminated rectangular sandwich plate. In-plane stress $\sigma_{x x}$ three-dimensional view of a quarter of the plate, and in-plane stress along the inplane axis direction $x$ evaluated at $(y, z)=(b / 2,+h / 2)$, for single and multi-models

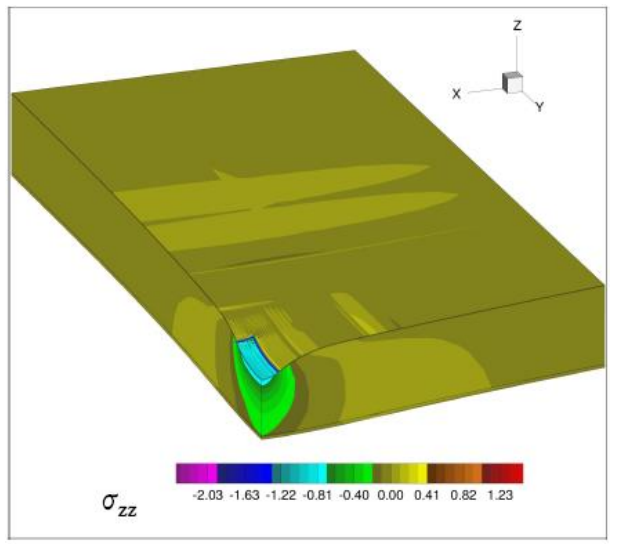

a) $L W 4$

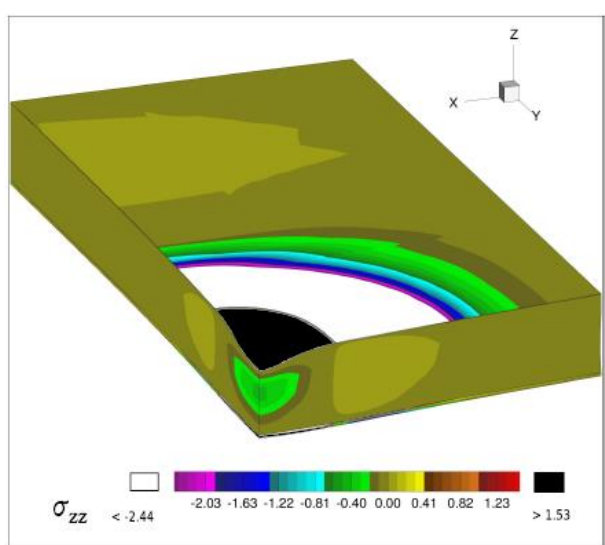

b) ET4

Fig. 20 Asymmetrically laminated rectangular sandwich plate, three-dimensional view of a quarter of the plate. Transverse normal stress $\sigma_{z z}$ for single models 
Multi-model Case $A$ and Case $B$ are shown in Figs. 21a and 21b, respectively. It is evident that the transverse normal stress is well represented in the LW4 zone only, close to the loaded zone.

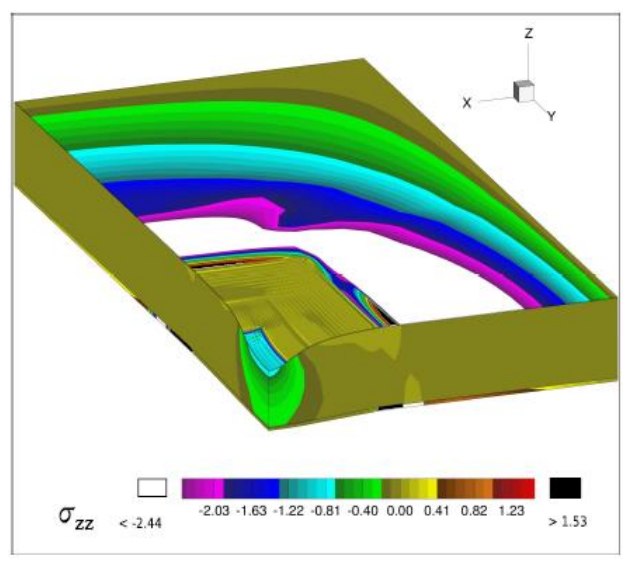

a) Case $\mathrm{A}$

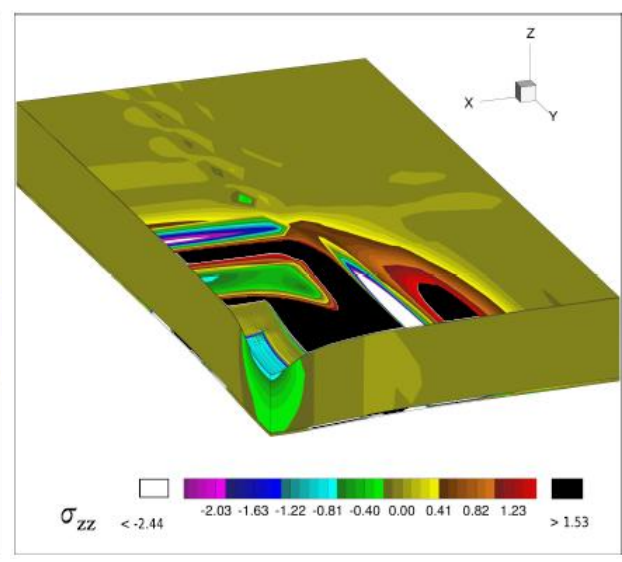

b) Case B

Fig. 21 Asymmetrically laminated rectangular sandwich plate, three-dimensional view of a quarter of the plate. Transverse normal stress $\sigma_{z z}$ for multi-models

\section{CONCLUSIONS}

A new simultaneous multi-model approach for a global/local analysis of composite and sandwich plates by means of a two-dimensional finite element with node-dependent kinematics has been introduced. The finite element governing equations are formulated in terms of fundamental nuclei, which are invariants of the theory approximation order. In this manner, the plate theory can vary within the same finite elements with no difficulties. No ad-hoc techniques and mathematical artifices are required to mix the fields coming from two different and kinematically incompatible adjacent zones, because the plate structural theory varies within the finite element itself; therefore, the same kinematics at the interface nodes between kinematically incompatible plate elements is enforced. The proposed methodology has been widely assessed in this paper by analyzing composite and sandwich plates and by comparison with analytical, FEM and 3D solid commercial solutions from the literature. Furthermore, it has been demonstrated that refined 2D models are able to detect complex strain-stress fields, in accordance with more cumbersome 3D models. Accurate results have been obtained in the refined part of the model with a significant reduction of the total number of degrees of freedom and, therefore, of the computational cost. Future developments will deal with the extension of this global/local methodology to hierarchical shell theories and to the Reissner Mixed Variational Theorem (RMVT). 


\section{REFERENCES}

1. Koiter, W.T., 1970, On the foundations of the linear theory of thin elastic shell, Proc. Kon. Nederl. Akad. Wetensch., 73, pp. 169-195.

2. Ciarlet, P.G., Gratie, L., 2005, Another approach to linear shell theory and a new proof of Korn's inequality on a surface, C. R. Acad. Sci. Paris, series I, 340(6), pp.471-478.

3. Reissner, E., Stavsky, Y., 1961, Bending and stretching of certain types of heterogeneous aelotropic elastic plates, Journal of Applied Mechanics, 28, pp. 402-408.

4. Reissner, E., 1945, The effect of transverse shear deformation on the bending of elastic plates, Journal of Applied Mechanics, 12(2), pp. 69-77.

5. Mindlin, R.D., 1951, Influence of rotary inertia and shear flexural motion of isotropic, elastic plates, Journal of Applied Mechanics, 18, pp. 31-38.

6. Kant, T., Owen, D.R.J., Zienkiewicz, O.C., 1982, Refined higher order $C^{0}$ plate bending element, Computer and Structures, 15, pp. 177-183.

7. Kant, T., Kommineni, J.R., 1989, Large amplitude free vibration analysis of cross-ply composite and sandwich laminates with a refined theory and $C^{0}$ finite elements, Computer and Structures, 50, pp. 123-134.

8. Reddy, J.N., 1997, Mechanics of Laminated Composite Plates and Shells, Theory and Analysis, CRC Press, New York, London, Tokyo.

9. Palazotto, A.N., Dennis, S.T., 1992, Nonlinear analysis of shell structures, AIAA Series, Washington.

10. Noor, A.K., Burton, W.S., 1990, Assessment of computational models for multi-layered composite shells, Applied Mechanics Review, 43, pp.67-97.

11. Reddy, J.N., 1993, An evaluation of equivalent-single-layer and layerwise theories of composite laminates, Composite Structures, 25, pp. 21-35.

12. Mawenya A.S., Davies, J.D., 1974, Finite element bending analysis of multilayer plates, Journal for Numerical Methods in Engineering, 8, pp. 215-225.

13. Rammerstorfer, F.G., Dorninger, K., Starlinger, A., 1992, Composite and sandwich shells, Nonlinear Analysis of Shells by Finite Elements, 328, pp. 131-194

14. Bank, R.E., 1983, The efficient implementation of local mesh refinement algorithms, in Babuska, I., Chandra, J., Flaherty, J.E. (eds.), Adaptive Computational Methods for Partial Differential Equations, SIAM, Philadelphia.

15. Szabo, B.A., Babuska, I., 1991, Finite Element Analysis, John Wiley \& Sons, New York, Toronto.

16. Bathe, K.J., 1996, Finite Element Procedures, Prentice Hall, New Jersey.

17. Thompson, D.M., Griffin, O.H.Jr., 1990, 2-D to 3-D Global/Local Finite Element Analysis of Cross-Ply Composite Laminates, Journal of Reinforced Plastics and Composites, 9, pp. 492-502.

18. Mao, K.M., Sun, C.T., 1991, A Refined Global-Local Finite Element Analysis Method, International Journal for Numerical Methods in Engineering, 32, pp.29-43, 1991.

19. Whitcomb, J.D., Woo, K., 1993, Application of iterative global/local finite element analysis. part 1: linear analysis, Communications in Numerical Methods in Engineering, 9(9), pp.745-756.

20. Whitcomb, J.D., Woo, K., 1993, Application of iterative global/local finite element analysis. part 2: geometrically non-linear analysis, Communications in Numerical Methods in Engineering, 9(9), pp. 757-766.

21. Wang, A.S.D., Crossman, F.W., 1978, Calculation of Edge Stresses in Multi-Layer by Sub-Structuring, Journal of Composite Materials, 12, pp.76-83.

22. Pagano, N. J., Soni, S.R., 1983, Global-local laminate variational model, International Journal of Solids and Structures, 19(3), pp. 207-228.

23. Jones, R., Callinan, R., Teh, K.K., Brown, K.C., 1984, Analysis of Multi-Layer Laminates Using ThreeDimensional Super Elements, International Journal for Numerical Methods in Engineering, 20(3), pp. 583-587.

24. Pagani, A., Valvano, S., Carrera, E., 2017, Analysis of laminated composites and sandwich structures by variable-kinematic MITC9 plate elements, Journal of Sandwich Structures and Materials, doi: $10.1177 / 1099636216650988$

25. Carrera, E., Pagani, A., Valvano, S., 2017, Shell elements with through-the-thickness variable kinematics for the analysis of laminated composite and sandwich structures, Composites Part B, 111, pp. 294-314.

26. Carrera, E., Valvano, S., 2017, A variable kinematic shell formulation applied to thermal stress of laminated structures, Journal of Thermal Stresses, doi: 10.1080/01495739.2016.1253439.

27. Brezzi F., Marini, L.D., 2005, The three-field formulation for elasticity problems, GAMM Mitteilungen, 28, pp. 124-153.

28. Carrera, E., Pagani, A., Petrolo, M., 2013, Use of Lagrange multipliers to combine $1 D$ variable kinematic finite elements, Computers and Structures, 129, pp. 194-206. 
29. Carrera, E., Pagani, A., 2013, Analysis of reinforced and thin-walled structures by multi-line refined 1D/beam models, International Journal of Mechanical Sciences, 75, pp. 278-287.

30. Carrera E., Pagani, A., 2014, Multi-line enhanced beam model for the analysis of laminated composite structures, Composites Part B, 57, pp. 112-119.

31. Dhia, H.B., 1998, Multiscale mechanical problems: the Arlequin method, Comptes Rendus de l'Academie des Sciences Series IIB Mechanics Physics Astronomy, 326(12), pp. 899-904.

32. Dhia, H.B., 1999, Numerical modelling of multiscale problems: the Arlequin method, CD Proceedings of ECCM'99, Munchen.

33. Dhia, H.B., 2008, Further insights by theoretical investigations of the multiscale Arlequin method, International Journal for Multiscale Computational Engineering, 6(3), pp. 215-232.

34. Dhia, H.B., The Arlequin method as a flexible engineering tool, International Journal for Numerical Methods in Engineering, 62(11), pp. 1442-1462.

35. Hu, H., Belouettar, S., Potier-Ferry, M., Daya, E.M., 2008, Multi-scale modelling of sandwich structures using the Arlequin method. Part I: linear modelling. Finite Elements in Analysis and Design, 45(1), pp. 37-51.

36. Hu, H., Belouettar, S., Potier-Ferry, M., Daya, E.M., Makradi, A., 2010, Multi-scale nonlinear modelling of sandwich structures using the Arlequin method, Finite Elements in Analysis and Design, 92(2), pp. 515-522.

37. Biscani, F., Giunta, G., Belouettar, S., Carrera, E., Hu, H., 2011, Variable kinematic beam elements coupled via Arlequin method, Composite Structures, 93(2), pp. 697-708.

38. Biscani, F., Giunta, G., Belouettar, S., Carrera, E., Hu, H., 2012, Variable kinematic plate elements coupled via Arlequin method, International Journal for Numerical Methods in Engineering, 91, pp.1264-1290.

39. Reddy, J.N., Robbins, D.H., 1994, Theories and computational models for composite laminates, Applied Mechanics Review, 47, pp. 147-165.

40. Reddy, J.N., 1997, Mechanics of Laminated Composite Plates - Theory and Analysis, CRC Press, Boca Raton.

41. Fish, J., 1992, The s-version of the finite element method, Computers and Structures, 43(3), pp. 539-547.

42. Fish, J., Markolefas, S., 1993, Adaptive s-method for linear elastostatics, Computer Methods in Applied Mechanics and Engineering, 103, pp. 363-396.

43. Wenzel, C., Vidal, P., D’Ottavio, M., Polit, O., 2014, Coupling of heterogeneous kinematics and Finite Element approximations applied to composite beam structures, Composite Structures, 116, pp. 177-192.

44. Carrera, E., Pagani, A., Valvano, S., 2017, Multilayered plate elements accounting for refined theories and nodedependent kinematics. Composites Part B, 114:189-210, 2017.

45. Bathe, K.J., Dvorkin, E., 1986, A formulation of general shell elements - the use of mixed interpolation of tensorial components, International Journal for Numerical Methods in Engineering, 22, pp. 697-722.

46. Bathe, K.J., Brezzi, F., 1987, A simplified analysis of two plate bending elements-the MITC4 and MITC9 elements, in Pande,G.N. et al. (eds.), Numerical Methods in Engineering: Theory and Applications, Martinus Nijhoff Publishers, Dordrecht.

47. Bathe, K.J., Brezzi, F., Cho, S.W., 1989, The MITC7 and MITC9 plate bending elements, Computers and Structures, 32(3-4), pp. 797-814.

48. Bucalem, M.L., Dvorkin, E., 1993, Higher-order MITC general shell elements, International Journal for Numerical Methods in Engineering, 36, pp. 3729-3754.

49. Carrera, E., Cinefra, M., Petrolo M, Zappino, E., 2014, Finite Element Analysis of Structures through Unified Formulation, John Wiley \& Sons, United Kingdom.

50. Carrera, E., Brischetto, S., 2008, Analysis of thickness locking in classical, refined and mixed multilayered plate theories, Composite Structures, 82, pp. 549-562.

51. Carrera, E., 2003, Theories and finite elements for multilayered plates and shells: a unified compact formulation with numerical assessment and benchmarking, Archives of Computational Methods in Engineering, 10(3), pp. 215-296.

52. Carrera, E., 1999, Multilayered shell theories accounting for layerwise mixed description, Part 1: governing equations, AIAA Journal, 37(9), pp. 1107-1116, 1999.

53. Carrera, E., 1999, Multilayered shell theories accounting for layerwise mixed description, Part 2: numerical evaluations. AIAA Journal, 37(9), pp. 1117-1124, 1999.

54. Cinefra, M., Valvano, S., Carrera, E., 2015, Heat conduction and Thermal Stress Analysis of laminated composites by a variable kinematic MITC9 shell element. Curved and Layered Structures, 1, pp. 301-320.

55. Cinefra, M., S. Valvano, Carrera, E., 2016, Thermal stress analysis of laminated structures by a variable kinematic MITC9 shell element. Journal of Thermal Stresses, 39(2), pp. 121-141.

56. Cinefra, M., Carrera, E., Valvano, S., 2015, Variable kinematic shell elements for the analysis of electromechanical problems, Mechanics of Advanced Materials and Structures, 22(1-2), pp. 77-106. 
57. Cinefra, M., Valvano, S., Carrera, E., 2015, A layer-wise MITC9 finite element for the free-vibration analysis of plates with piezo-patches, International Journal of Smart and Nano Materials, 6(2), pp. 85- 104.

58. Carrera, E., 2003, Historical review of zig-zag theories for multilayered plates and shells, Applied Mechanics Reviews, 56, pp. 287-308.

59. Carrera, E., On the use of the Murakami's zig-zag function in the modeling of layered plates and shells. Computers and Structures, 82, pp. 541-554.

60. Carrera, E., 1998, Mixed layer-wise models for multilayered plates analysis, Composite Structures, 43, pp. $57-70$.

61. Carrera, E., 1998, Evaluation of Layerwise Mixed Theories for Laminated Plates Analysis, Applied Mechanics Reviews, 36(5), pp. 830-839.

62. Lekhnitskii, S.G., 1968, Anisotropic Plates, Gordon \& Branch, New York.

63. Nguyen, S.H., Surana, K.S., 1990, Two-dimensional curved beam element with higher-order hierarchical transverse approximation for laminated composites, Computers \& Structures, 36, pp. 499-511.

64. Davalos, J.F. Kim, Y., Barbero, E.J., 1994, Analysis of laminated beams with a layerwise constant shear theory, Computers and Structures, 28, pp. 241-253.

65. Xiaoshan Lin, Y.Z., 2011, A novel one-dimensional two-node shear-flexible layered composite beam element, Finite Elements in Analysis and Design, 47, pp. 676-682.

66. Vo, T.P., Thai, H.T., 2012, Static behavior of composite beams using various refined shear deformation theories, Computers and Structures, 94, pp. 2513-2522.

67. Meyer-Piening, H.R., 2000, Experiences with 'exact' linear sandwich beam and plate analyses regarding bending, instability and frequency investigations, In Meyer-Piening, H.R., Zenkert, D. (eds.), Proceedings of the Fifth International Conference on Sandwich Constructions, vol. 1, pp 37-48, EMAS Publishing, Zurich.

68. Carrera, E, Ciuffreda, A, 2005, Bending of composites and sandwich plates subjected to localized lateral loadings: a comparison of various theories, Composite Structures, 68, pp. 185-202.

69. Carrera, E., Demasi, L., 2003, Two benchmarks to assess two-dimensional theories of sandwich, composite plates, AIAA Journal, 41(7), pp. 1356-1362. 